\title{
Extension of moment projection method to the fragmentation process
}

\author{
Shaohua $\mathrm{Wu}^{\mathrm{a}}$, Edward K. Y. Yapp ${ }^{\mathrm{b}}$, Jethro Akroyd ${ }^{\mathrm{b}}$, Sebastian Mosbach ${ }^{\mathrm{b}}$, \\ Rong $\mathrm{Xu}^{\mathrm{c}}$, Wenming Yang ${ }^{\mathrm{a}}$, Markus Kraft*b,c \\ ${ }^{a}$ Department of Mechanical Engineering, National University of Singapore, \\ Engineering Block EA, Engineering Drive 1, Singapore, 117576 \\ ${ }^{b}$ Department of Chemical Engineering and Biotechnology, University of Cambridge, \\ New Museums Site, Pembroke Street, Cambridge, CB2 3RA United Kingdom \\ ${ }^{c}$ School of Chemical and Biomedical Engineering, Nanyang Technological University, \\ 62 Nanyang Drive, Singapore, 637459 \\ corresponding author* \\ E-mail:mk306@cam.ac.uk
}

\section{Abstract}

The method of moments is a simple but efficient method of solving the population balance equation which describes particle dynamics. Recently, the moment projection method (MPM) was proposed and validated for particle inception, coagulation, growth and, more importantly, shrinkage; here the method is extended to include the fragmentation process. The performance of MPM is tested for 13 different test cases for different fragmentation kernels, fragment distribution functions and initial conditions. Comparisons are made with the quadrature method of moments (QMOM), hybrid method of moments (HMOM) and a high-precision stochastic solution calculated using the established direct simulation algorithm (DSA) and advantages of MPM are drawn.

Keywords: Fragmentation, breakage, method of moments, population balance, moment projection method, particulate systems 
1

2

\section{Introduction}

Fragmentation (also referred to as breakage) is a process by which particles break into two or more fragments leading to an increase in the number of particles [1]. For this reason it plays an important role in a number of chemical processes [2]. In fluidised-bed combustion, the rate of fragmentation during particle burnout influences the overall burning rate of single coal particles [3]. Arguably, in practical combustion systems, predicting particle destruction can be as important as predicting particle formation and growth. It is found in Ref. [4] that the inclusion of fragmentation improved model predictions of soot particle size distributions (PSDs) from a diesel engine.

The evolution of the PSD with time is described by the population balance equation (PBE) with mechanisms which modify the particles such as inception, coagulation (otherwise known as aggregation), growth, and shrinkage where particles reduce in mass and are eventually removed from the system [5-7]. In Ref. [8] the PBE for a particulate system undergoing fragmentation is studied and it is found that the PSD obeys a first-order linear ordinary integro-differential equation. The complexity of the equation depends on the fragmentation kernel and fragment distribution function, and analytical solutions only exist for certain restrictive cases.

A number of methods have been proposed to solve these types of equations which can be broadly classified as: method of moments (MOM) (see, e.g., Refs. [2, 4-7, 9-21]), sectional method (see, e.g., Refs. [1, 9, 22-29]) and stochastic method (see, e.g., Refs. [11, 30-35]). These methods often encompass a trade-off between physical detail and computational efficiency. In the stochastic method the particle population is represented by an en- 
semble of stochastic particles and the particle processes are treated probabilistically [36]. The stochastic solution has been proven to converge to the deterministic solution of the PBE [33]. The method easily allows a highly detailed particle description; however, under certain conditions, the computational time [34] and memory requirement [35] can be intractable. Sectional methods divide the mass range into a finite number of sections [24]. The PSD within each section evolves according to a ordinary differential equation which can be solved by standard solvers (see, e.g., Refs. [25-28]). The computational time rapidly scales with the number of internal coordinates tracked and the number of sections required to achieve convergence [29].

When the PBE is written in terms of one or two internal coordinates, MOM is a particularly attractive option for its computational efficiency [13, 14]. The PBE is rewritten in terms of moments and one solves for just the first few moments which are usually sufficient for most practical applications [37]. Development of MOM for the fragmentation/breakage process is a particularly active field of research (see, e.g., Refs. $[7,15]$ ). In Ref. [7] the hybrid method of moments (HMOM) [6] is extended to model the fragmentation of soot aggregates in laminar flames. HMOM combines the numerical ease of the method of moments with interpolative closure (MOMIC) [37] and the accuracy of the direct quadrature method of moments (DQMOM) [21] with a source term for the smallest particles based on the negative infinity moment. The production of the smallest particles was assumed to be proportional to the mass lost from the large particles. Symmetric fragmentation was assumed where one particle fragments into two identical particles. In this paper we test HMOM, albeit a spherical particle description, for both 
symmetric fragmentation and erosion distribution functions.

Another widely used moment method that has been used to address breakage is the quadrature method of moments (QMOM) [17-20] where the PSD is approximated by a weighted summation of Dirac delta functions. The performance of QMOM for simultaneous aggregation and breakage problems with different combinations of aggregation and breakage kernels, fragment distribution functions and initial conditions has been investigated in Ref. [20]. A quadrature approximation with two nodes was found to be sufficiently accurate for most cases except for symmetric fragmentation with a constant kernel and erosion with a size-dependent kernel. Increasing the number of nodes did not help in decreasing the error in some cases. However, across all cases aggregation was dominant. The accuracy of QMOM in treating pure breakage problems or where breakage is the dominant process has not been addressed yet. This paper will be a step in this direction.

In Ref. [38] a finite-size domain complete set of trial functions method of moments (FCMOM) is proposed which uses a series of Legendre polynomials to reconstruct the PSD, thus closing the moment equations. However, because only a finite number of polynomials can be determined, certain values of the reconstructed PSD can be negative [39]. An alternative method is the extended quadrature method of moments (EQMOM) where a set of non-negative continuous kernel density functions such as gamma, beta and lognormal functions is adopted to approximate the PSD. In terms of the reconstructed PSD this method can achieve very high accuracy and is able to handle the shrinkage problem. However, information about the shape of the PSD is needed a priori to select a suitable kernel density function. Both 
FCMOM and EQMOM are focused on the reconstruction of the PSD while for most practical applications only the first few moments are needed.

Recently, a moment projection method (MPM) [5] was developed to address the shrinkage of particles. It directly solves the moment transport equation and tracks the number of the smallest particles using the algorithm by Blumstein and Wheeler [40]. A similar algorithm for solving the Gauss-Radau quadrature is given by Golub [41, 42]. In both algorithms the derivation is given in terms of orthogonal polynomials which is straightforward and can be easily modified to treat the cases in which zero, one or two particle mass classes are fixed. The ability of MPM to simulate shrinkage problems was investigated and the advantages of the method was highlighted. To be able to model fragmentation accurately one has to be able to model the number of the smallest particles accurately which are formed under strong fragmentation. Therefore, fragmentation is a natural extension of MPM.

For quadrature-based moment methods a very important consideration is the realisability of the moment set [43]. Realisability is related to the existence of an underlying PSD that corresponds to a set of moments. The moments are linked to each other under complex mathematical relationships. If the numerical schemes do not preserve these relationships the set of moments can be unrealisable, i.e., no PSD can be described by such moments or they lead to unphysical distributions (e.g. negative weights and abscissas). The generation of unrealizable moments usually arises from the spatial transportation of moments [44]. Even if a suitable closure is established for the moment transport equation, numerical advection and diffusion schemes can still lead to unrealizable moment sets. This realisability problem can be 
avoided by properly designing the numerical schemes. For example, recently in Ref. [45] a high-order-volume-schemes for quadrature-based moment methods is introduced to guarantee the realisability of moments. The idea of the discretization scheme is to construct the moment flux terms through interpolation of the quadrature weights rather than the moments at the faces of the cells. By doing this the realisability problem can be prevented. Another scheme developed to preserve the realisability of moments can be found in Ref. [46] where the moments are not transported directly. Instead they use the canonical moments which are easy to control and guarantee the moment vector to stay in the moment space by transporting them separately. In light of realisability, here we restrict our attention to the moment closure method. The aim is to investigate the MPM error in isolation. Therefore we are simulating a spatially homogenous PBE with no moment advection and diffusion terms. The moments always remain realizable during the whole simulation time span. While for the application of MPM to spatially inhomogeneous systems, moments realizability can be guranteed by adopting the realizable finite-volume methods.

In this work, different types of fragmentation kernels, fragment distribution functions and initial conditions are imposed and the results are compared with QMOM, HMOM and a high-precision stochastic solution. Both QMOM and HMOM have the advantages of mathematical simplicity, numerical robustness and ease of implementation. The stochastic solution was obtained with 131,072 stochastic particles in a single run and is used as "exact" solution in this work. The paper is organized as follows. Section 2 presents the moment of methods for solving the PBE as well as the mathematical for- 
mulation and numerical algorithm of MPM. In Section 3 the performance of MPM is tested for different test cases and in Section 4 principal conclusions are summarised.

\section{Moment methods for population balance equations}

\subsection{Population balance equation}

A spatially homogeneous population of particles with a discrete-mass distribution is considered in this work. The smallest particles have mass $m_{1}$ and particles in the mass class $i$ have mass $m_{i}=i m_{1}$. The PBE governing the evolution of the distribution can be written as:

$$
\frac{\mathrm{d} N(i, t)}{\mathrm{d} t}=R(i, t)+W(i, t)+S(i, t)+G(i, t)+F(i, t), \quad i=1,2, \ldots, \infty
$$

where $N(i, t)$ is the number of particles in the mass class $i$ at time $t$ which we will refer to as $N_{i}$ from hereon. This is known as a particle number representation of the PSD. $R, W, S, G$ and $F$ are the inception, growth, shrinkage, coagulation and fragmentation terms, respectively. The specific functional forms used in this work are as follows: 


$$
\begin{aligned}
R(i=1, t) & =I_{m_{1}}, \\
W(i, t) & =K_{\mathrm{G}}(i-\delta) N_{i-\delta}-K_{\mathrm{G}}(i) N_{i}, \\
S(i, t) & =K_{\mathrm{Sk}}(i+\delta) N_{i+\delta}-K_{\mathrm{Sk}}(i) N_{i}, \\
G(i, t) & =\frac{1}{2} \sum_{j=1}^{i} K_{\mathrm{Cg}}(j, i-j) N_{j} N_{i-j}-\sum_{j=1}^{\infty} K_{\mathrm{Cg}}(i, j) N_{i} N_{j}, \\
F(i, t) & =\sum_{j=i}^{\infty} K_{\mathrm{Fg}}(j) P(i \mid j) N_{j}-K_{\mathrm{Fg}}(i) N_{i},
\end{aligned}
$$

where $I_{m_{1}}$ is the inception kernel which describes the rate of formation of the smallest particles. $K_{\mathrm{G}}$ and $K_{\mathrm{Sk}}$ are the growth and shrinkage kernels, respectively, where $\delta$ refers to the mass change in a single growth or shrinkage event which can be different. $K_{\mathrm{Cg}}$ is the coagulation kernel which describes the rate at which particles collide and stick together. Lastly, $K_{\mathrm{Fg}}$ is the fragmentation kernel which describes the frequency with which particles fragment and $P(i \mid j)$ is the fragment distribution function which represents the number of particles of mass class $i$ formed by the fragmentation of particles of mass class $j$.

The choice of fragmentation kernel and fragment distribution function are important because for certain combinations, "shattering" may occur [47, 48]. In a process analogous to gelation (but in the oppposite sense), a finite fraction of the mass shatters into an infinite number of particles of zero mass and for this reason mass is not conserved [49]. This usually occurs when the fragmentation rate increases as the particles become smaller. Note that self-similar solutions where the PSD does not vary with time are of special interest as the PSD is independent of initial conditions and most experimental 
systems evolve to the point where this behaviour is reached [50]. It is found in Ref. [51] that a self-similar PSD is achieved when the fragmentation kernel is of the power type and the fragment distribution function depends on the parent-daughter particle mass ratio.

Many different functional forms of the fragment distribution function have been proposed, however some physical constraints must be fulfilled [51, 52]:

$$
\begin{aligned}
P(i \mid j) & =0, \quad \text { for } i>j, \\
\sum_{i=1}^{j} i P(i \mid j) & =j .
\end{aligned}
$$

The first equation states that fragmentation can only lead to the formation of particles of mass class $i$ smaller than the parent particle mass class $j$, while the second equation is the conservation of mass where the total mass class of particles resulting from the breakup of a particle of mass class $j$ must be equal to $j$. In this work, we only consider binary fragmentation and the fragment distribution functions are reported in Table 1; a discussion of multiple fragmentation can be found in Ref. [51]. Symmetric fragmentation leads to the formation of two equal mass fragments, whereas in the case of erosion one fragment is of the smallest mass class $i=1$ while the other is of the mass class $i=j-1$.

\subsection{Moment equations}

As mentioned earlier, an efficient approach for solving the PBE is MOM where the PBE is transformed into a set of moment equations and integral quantities such as the total particle number and mass are computed. This is achieved by applying the definition, moment of order $k$ of the PSD 
Table 1: Fragmentation distribution functions.

\begin{tabular}{ll}
\hline Mechanism & $P(i \mid j)$ \\
\hline Symmetric fragmentation & $\begin{cases}2 & \text { if } i=j / 2 \\
0 & \text { otherwise }\end{cases}$ \\
Erosion & $\left\{\begin{array}{lll}1 & \text { if } i=1 \\
1 & \text { if } i=j-1 \\
0 & \text { otherwise }\end{array}\right.$ \\
\hline
\end{tabular}

$$
M_{k}=\sum_{i=1}^{\infty} i^{k} N_{i}, \quad k=0,1,2, \ldots,
$$

178

to Eq. (1), leading to

$$
\frac{\mathrm{d} M_{k}}{\mathrm{~d} t}=R_{k}(M)+G_{k}(M)+W_{k}(M)+S_{k}\left(M, N_{1}\right)+F_{k}\left(M, N_{1}\right),
$$

179 where 


$$
\begin{aligned}
R_{k}(M) & =m_{1}^{k} I_{m_{1}} \\
G_{k}(M) & =\frac{1}{2} \sum_{i=1}^{\infty} \sum_{j=1}^{i-1} i^{k} K_{\mathrm{Cg}}(j, i-j) N_{j} N_{i-j}-\sum_{i=1}^{\infty} \sum_{j=1}^{\infty} i^{k} K_{\mathrm{Cg}}(i, j) N_{i} N_{j} \\
W_{k}(M) & =\sum_{i=1}^{\infty} K_{\mathrm{G}}(i-\delta) i^{k} N_{i-\delta}-\sum_{i=1}^{\infty} K_{\mathrm{G}}(i) i^{k} N_{i} \\
S_{k}\left(M, N_{1}\right) & =\sum_{i=1}^{\infty} K_{\mathrm{Sk}}(i+\delta) i^{k} N_{i+\delta}-\sum_{i=1}^{\infty} K_{\mathrm{Sk}}(i) i^{k} N_{i} \\
F_{k}\left(M, N_{1}\right) & =\sum_{j=1}^{\infty} \sum_{i=1}^{j} K_{\mathrm{Fg}}(j) i^{k} P(i \mid j) N_{j}-\sum_{i=1}^{\infty} K_{\mathrm{Fg}}(i) i^{k} N_{i}
\end{aligned}
$$

Evaluation of the moment source terms depends on the kernel function $K$. It is assumed that when the smallest particles shrink they are removed from the system, while for the fragmentation process the smallest particles are unbreakable. Depending on the specific kernels used the shrinkage and fragmentation source terms $S_{k}$ and $F_{k}$ can depend on the number of the smallest particles $N_{1}$. These will be specified later. Where realistic kernels are used, fractional- or even negative-order moments are encountered [14]. Therefore, the mathematical difficulty of MOM lies in obtaining closure for these moment source terms using a finite set of moments. This requires either a priori assumptions about the shape of the PSD or a suitable closure scheme. One of the more widely used closure methods is MOMIC [37] where closure is accomplished by Langrange polynomial interpolation of the logarithm of the whole-order moments whose values are available at each integration step of Eq. (10). By separating interpolation for positive- and negative-order mo- 
ments, MOMIC shows very high accuracy in the treatment of mono-modal PSDs undergoing growth and coagulation and satisfactory accuracy for bimodal PSDs formed under persistent nucleation [6]. However, MOMIC cannot handle shrinkage as it does not track $N_{1}$. Likewise, it cannot rigorously treat fragmentation especially erosion where a large number of particles accumulate in the smallest particle mass class.

\subsection{Moment projection method}

The mathematical formulation and numerical algorithm of MPM have already been presented in Ref. [5], however, pertinent details are repeated here for the reader's convenience. In MPM, we approximate the true PSD by assuming that all particles are distributed into a finite number of particle mass classes. The $k$-th order moment of the approximated PSD can then be expressed as:

$$
\widetilde{M}_{k}=\alpha_{1}^{k} \widetilde{N}_{\alpha_{1}}+\sum_{j=2}^{N_{\mathrm{p}}} \alpha_{j}^{k} \widetilde{N}_{\alpha_{j}}, \quad k=0, \ldots, 2 N_{\mathrm{p}}-2
$$

where $\alpha_{j}$ is the particle mass, $\widetilde{N}_{\alpha_{j}}$ is the number of particles of the mass $\alpha_{j}$, and $N_{\mathrm{p}}$ is the number of particle masses used to represent the PSD. The symbol " $\sim$ " is used to indicate approximations of the corresponding quantity from the true PSD. $\alpha_{j}$ and $\widetilde{N}_{\alpha_{j}}$ are chosen such that the empirical moments are equal to the moments from the true PSD:

$$
\widetilde{M}_{k}=M_{k}
$$

Applying Eq. (17) to Eq. (10), we obtain: 


$$
\frac{\mathrm{d} \widetilde{M}_{k}}{\mathrm{~d} t}=R_{k}(\widetilde{M})+G_{k}(\widetilde{M})+W_{k}(\widetilde{M})+S_{k}\left(\widetilde{M}, N_{1}\right)+F_{k}\left(\widetilde{M}, N_{1}\right)
$$

213

To evaluate the boundary flux term $N_{1}$ present in the shrinkage and fragmentation terms, we fix the first particle mass to be equal to the smallest particle mass of the true PSD: $\alpha_{1}=m_{1}$. Therefore, $\widetilde{N}_{\alpha_{1}}$ is an approximation of the number of the smallest particle which allows us to express Eq. (18) as:

$$
\frac{\mathrm{d} \widetilde{M}_{k}}{\mathrm{~d} t}=R_{k}(\widetilde{M})+G_{k}(\widetilde{M})+W_{k}(\widetilde{M})+S_{k}\left(\widetilde{M}, \widetilde{N}_{\alpha_{1}}\right)+F_{k}\left(\widetilde{M}, \widetilde{N}_{\alpha_{1}}\right)
$$

As can be seen from Eq. (19), $\widetilde{M}_{k}$ is directly evaluated from the moment transport equation which allows us to take advantage of MOMIC when realistic kernels are used. However, this introduces an interpolation error. The aim here is to investigate the MPM error in isolation, therefore constant kernels are adopted:

$$
\begin{aligned}
& R_{k}(\widetilde{M})=m_{1}^{k} I_{m_{1}}, \quad k=0, \ldots, 2 N_{\mathrm{p}}-2, \\
& G_{k}(\widetilde{M})= \begin{cases}-\frac{1}{2} K_{\mathrm{Cg}} \widetilde{M}_{0}^{2}, & k=0, \\
0, & k=1, \\
\frac{1}{2} K_{\mathrm{Cg}} \sum_{r=1}^{k-1}\left(\begin{array}{l}
k \\
r
\end{array}\right) \widetilde{M}_{r} \widetilde{M}_{k-r}, & k=2, \ldots, 2 N_{\mathrm{p}}-2,\end{cases} \\
& W_{k}(\widetilde{M})= \begin{cases}0, & k=0, \\
K_{\mathrm{G}} \sum_{r=1}^{k}\left(\begin{array}{l}
k \\
r
\end{array}\right) \delta^{r} \widetilde{M}_{k-r}, & k=1, \ldots, 2 N_{\mathrm{p}}-2,\end{cases}
\end{aligned}
$$




$$
S_{k}\left(\widetilde{M}, \widetilde{N}_{\alpha_{1}}\right)=\left\{\begin{array}{lc}
-K_{\mathrm{Sk}} \widetilde{N}_{\alpha_{1}}, & k=0, \\
K_{\mathrm{Sk}} \sum_{r=1}^{k}\left(\begin{array}{l}
k \\
r
\end{array}\right)(-\delta)^{r} \widetilde{M}_{k-r}, k=2, \ldots, 2 N_{\mathrm{p}}-2 .
\end{array}\right.
$$

$F_{k}\left(\widetilde{M}, \widetilde{N}_{\alpha_{1}}\right)= \begin{cases}K_{\mathrm{Fg}}\left(\widetilde{M}_{0}-\widetilde{N}_{\alpha_{1}}\right), & k=0 \\ 0, & k=1, \\ K_{\mathrm{Fg}} \alpha_{1}^{k} \widetilde{M}_{0}+K_{\mathrm{Fg}} \sum_{r=1}^{k}\left(\begin{array}{l}k \\ r\end{array}\right)\left(-\alpha_{1}\right)^{r} \widetilde{M}_{k-r}, & k=2, \ldots, 2 N_{\mathrm{p}}-2 .\end{cases}$

225

The fragmentation source term depends on the fragment distribution function. For symmetric fragmentation it is:

$$
F_{k}\left(\widetilde{M}, \widetilde{N}_{\alpha_{1}}\right)= \begin{cases}K_{\mathrm{Fg}}\left(\widetilde{M}_{0}-\widetilde{N}_{\alpha_{1}}\right), & k=0, \\ 0, & k=1, \\ K_{\mathrm{Fg}}\left(2^{1-k}-1\right)\left(\widetilde{M}_{k}-\alpha_{1}^{k} \widetilde{N}_{\alpha_{1}}\right), & k=2, \ldots, 2 N_{\mathrm{p}}-2,\end{cases}
$$

and for erosion:

In Ref. [30] a fragmentation kernel with a linear dependence on particle mass is used to study the wet granulation of particles. Since the fragmentation moment source term can be evaluated based on the whole-moments, we also investigate the same fragmentation kernel which for symmetric fragmentation is: 


$$
F_{k}\left(\widetilde{M}, \widetilde{N}_{\alpha_{1}}\right)= \begin{cases}K_{\mathrm{Fg}}\left(\widetilde{M}_{1}-\alpha_{1} \widetilde{N}_{\alpha_{1}}\right), & k=0, \\ 0, & k=1, \\ K_{\mathrm{Fg}}\left(2^{1-k}-1\right)\left(\widetilde{M}_{k+1}-\alpha_{1}^{k+1} \widetilde{N}_{\alpha_{1}}\right), & k=2, \ldots, 2 N_{\mathrm{p}}-2,\end{cases}
$$

230 and for erosion:

$$
F_{k}\left(\widetilde{M}, \widetilde{N}_{\alpha_{1}}\right)= \begin{cases}K_{\mathrm{Fg}}\left(\widetilde{M}_{1}-\alpha_{1} \widetilde{N}_{\alpha_{1}}\right), & k=0, \\
0, & k=1, \\
K_{\mathrm{Fg}} \alpha_{1}^{k} \widetilde{M}_{1}+K_{\mathrm{Fg}} \sum_{r=1}^{k}\left(\begin{array}{l}
k \\
r
\end{array}\right)\left(-\alpha_{1}\right)^{r} \widetilde{M}_{k-r+1}, & k=2, \ldots, 2 N_{\mathrm{p}}-2 .\end{cases}
$$

${ }_{231}$ The challenge now is determining $\alpha_{j}$ and $\widetilde{N}_{\alpha_{j}}$ such that Eq. (17) is true ${ }_{232}$ while fulfilling the requirement that $\widetilde{N}_{\alpha_{1}} \approx N_{1}$ to close the moment source 233 terms due to shrinkage and fragmentation. This can be achieved using the ${ }_{234}$ Blumstein and Wheeler algorithm [40] which can be found in Appendix 2. 235 The numerical procedure of MPM is summarized in Algorithm 1. 
Algorithm 1: Moment projection method algorithm.

Input: Moments of the PSD $M_{k}\left(t_{0}\right)$ for $k=0, \ldots, 2 N_{\mathrm{p}}-2$ or the PSD itself

$N\left(i, t_{0}\right)$ for $i=1, \ldots, \infty$ at initial time $t_{0}$; final time $t_{\mathrm{f}}$.

Output: Empirical moments of the PSD $\widetilde{M}_{k}\left(t_{\mathrm{f}}\right)$ for $k=0, \ldots, 2 N_{\mathrm{p}}-2$ at final time $t_{\mathrm{f}}$ where $N_{\mathrm{p}}$ is the number of particle masses used to approximate the PSD.

Calculate the moments of the true PSD using Eq. (9):

$$
M_{k}\left(t_{0}\right)=\sum_{i=1}^{\infty} i^{k} N\left(i, t_{0}\right), \quad k=0, \ldots, 2 N_{\mathrm{p}}-2
$$

For $\widetilde{M}_{k}=M_{k}$, solve Eq. (16) for $\widetilde{N}_{\alpha_{1}}\left(\alpha_{1}\right.$ is fixed) and $\alpha_{j}$ and $\widetilde{N}_{\alpha_{j}}$

$\left(j=2, \ldots, N_{\mathrm{p}}\right)$ using Algorithm 2 :

$$
\widetilde{M}_{k}\left(t_{0}\right)=\alpha_{1}^{k} \widetilde{N}_{\alpha_{1}}\left(t_{0}\right)+\sum_{j=2}^{N_{\mathrm{p}}} \alpha_{j}^{k} \widetilde{N}_{\alpha_{j}}\left(t_{0}\right), \quad k=0, \ldots, 2 N_{\mathrm{p}}-2 .
$$

$t \longleftarrow t_{0}, \widetilde{M}_{k}(t) \longleftarrow \widetilde{M}_{k}\left(t_{0}\right)$

while $t<t_{f}$ do

Integrate Eq. (19) over the time interval $\left[t_{i}, t_{i}+h\right]$ using a fouth-order

Runge-Kutta method:

$$
\frac{\mathrm{d} \widetilde{M}_{k}}{\mathrm{~d} t}=R_{k}(\widetilde{M})+G_{k}(\widetilde{M})+W_{k}(\widetilde{M})+S_{k}\left(\widetilde{M}, \widetilde{N}_{\alpha_{1}}\right)+F_{k}\left(\widetilde{M}, \widetilde{N}_{\alpha_{1}}\right)
$$

with initial condition:

$$
\left(\begin{array}{c}
\widetilde{M}_{k}\left(t_{i}\right) \\
\widetilde{N}_{\alpha_{1}}\left(t_{i}\right)
\end{array}\right)=\left(\begin{array}{c}
\widetilde{M}_{k, i} \\
\widetilde{N}_{\alpha_{1}, i}
\end{array}\right)
$$

where $R_{k}(\widetilde{M}), G_{k}(\widetilde{M}), W_{k}(\widetilde{M})$ and $S_{k}\left(\widetilde{M}, \widetilde{N}_{\alpha_{1}}\right)$ are given by Eqs. (20), (21), (22) and (23), respectively. The form of $F_{k}\left(\widetilde{M}, \widetilde{N}_{\alpha_{1}}\right)$ depends on the fragmentation kernel and fragment distribution function as given by Eqs. (24-27).

Use Blumstein algorithm to update $\alpha_{j}$ and $\widetilde{N}_{\alpha_{j}}$, and assign solution at $t_{i+1}=t_{i}+h:$

$i \longleftarrow i+1$

$$
\left(\begin{array}{c}
\widetilde{M}_{k, i+1} \\
\widetilde{N}_{\alpha_{1}, i+1}
\end{array}\right)_{16} \leftarrow\left(\begin{array}{l}
\widetilde{M}_{k}\left(t_{i}+h\right) \\
\widetilde{N}_{\alpha_{1}}\left(t_{i}+h\right)
\end{array}\right)
$$




\section{Numerical results}

As the focus of this paper is to test MPM for the process of fragmentation, we devise a number of test cases which can be classified into the following three categories: (1) pure fragmentation, (2) simultaneous coagulation and fragmentation, and (3) all particle processes combined (inception, growth, coagulation, shrinkage and fragmentation). It is assumed that the smallest particles are unbreakable, i.e., $K_{\mathrm{Fg}}(i=1)=0$. Log-normal, unimodal and parabolic PSDs are supplied as the initial condition.

Numerical results are compared to those from HMOM, QMOM and a high-precision stochastic solution calculated using the direct simulation algorithm (DSA). HMOM was originally developed for bivariate PBEs $[6,7]$. We modify this method so that it is applicable to monovariate PBEs. Details on the modifications made, with a focus on the fragmentation process, can be found in Appendix B.

\subsection{Pure fragmentation}

The fragmentation kernels, fragment distribution functions and initial conditions used to test pure fragmentation are reported in Table 2 .

For Case 1 particles undergo symmetric fragmentation with a constant kernel; a log-normal distribution is supplied as the initial condition. The moment transport equation with the fragmentation moment source term in Eq. (24) is solved. The particle masses $\alpha_{j}$ and the corresponding number of particles $\widetilde{N}_{\alpha_{j}}$ describing the evolution of the moments of the PSD are computed using MPM and are shown in Fig. 1. Four particle masses are used to approximate the PSD. $\alpha_{j}(j=2,3,4)$ decrease as particles fragment 
Table 2: Cases used for the comparison of pure fragmentation.

\begin{tabular}{|c|c|c|c|c|}
\hline Case & $K_{\mathrm{Fg}}(i)$ & & $P(i \mid j)$ & $N_{i}(t=0)$ \\
\hline 1 & 0 & $i=1$ & Symmetric & $N_{i}=10^{5} \exp \left(-\left(\log \left(2^{i-1}\right)-\log (32)\right)^{2} / 0.05\right)$ \\
\hline & 20 & $i>1$ & fragmentation & $i=1, \ldots, 10$ \\
\hline 2 & $\begin{array}{l}0 \\
2 i\end{array}$ & $\begin{array}{l}i=1 \\
i>1\end{array}$ & Erosion & $N_{i}=100, i=30$ \\
\hline 3 & 0 & $i=1$ & Symmetric & $N_{i}=10^{5} \exp \left(-\left(\log \left(2^{i-1}\right)-\log (16)\right)^{2} / 0.05\right)$ \\
\hline & $0.2 i$ & $i>1$ & fragmentation & $i=1, \ldots, 10$ \\
\hline 4 & $\begin{array}{l}0 \\
0.2 i\end{array}$ & $\begin{array}{l}i=1 \\
i>1\end{array}$ & $\begin{array}{l}\text { Symmetric } \\
\text { fragmentation }\end{array}$ & $N_{i}=10000, i=256$ \\
\hline 5 & $\begin{array}{l}0 \\
2\end{array}$ & $\begin{array}{l}i=1 \\
i>1\end{array}$ & Erosion & $N_{i}=300 i-10 i^{2}, i=1, \ldots, 30$ \\
\hline 6 & $\begin{array}{l}0 \\
2\end{array}$ & $\begin{array}{l}i=1 \\
i>1\end{array}$ & Erosion & $\begin{array}{l}N_{i}=100 \exp \left(-(\log (i)-\log (25))^{2} / 0.05\right) \\
i=1, \ldots, 100\end{array}$ \\
\hline 7 & $\begin{array}{l}0 \\
2 i\end{array}$ & $\begin{array}{l}i=1 \\
i>1\end{array}$ & Erosion & $\begin{array}{l}N_{i}=100 \exp \left(-(\log (i)-\log (25))^{2} / 0.05\right) \\
i=1, \ldots, 100\end{array}$ \\
\hline
\end{tabular}

to form increasingly smaller particles. The number of particles of the largest mass $\widetilde{N}_{\alpha_{4}}$ decreases leading to an initial increase in $\widetilde{N}_{\alpha_{2}}$ and $\widetilde{N}_{\alpha_{3}}$ before also decreasing. $\widetilde{N}_{\alpha_{1}}$ increases and shows an asymptote at around $N=3.0 \times 10^{6}$ as particles of the smallest mass $m_{1}$ are formed which are assumed to not be able to fragment further.

To assess the accuracy of the moments calculated using MPM the following relative error metric is used: 

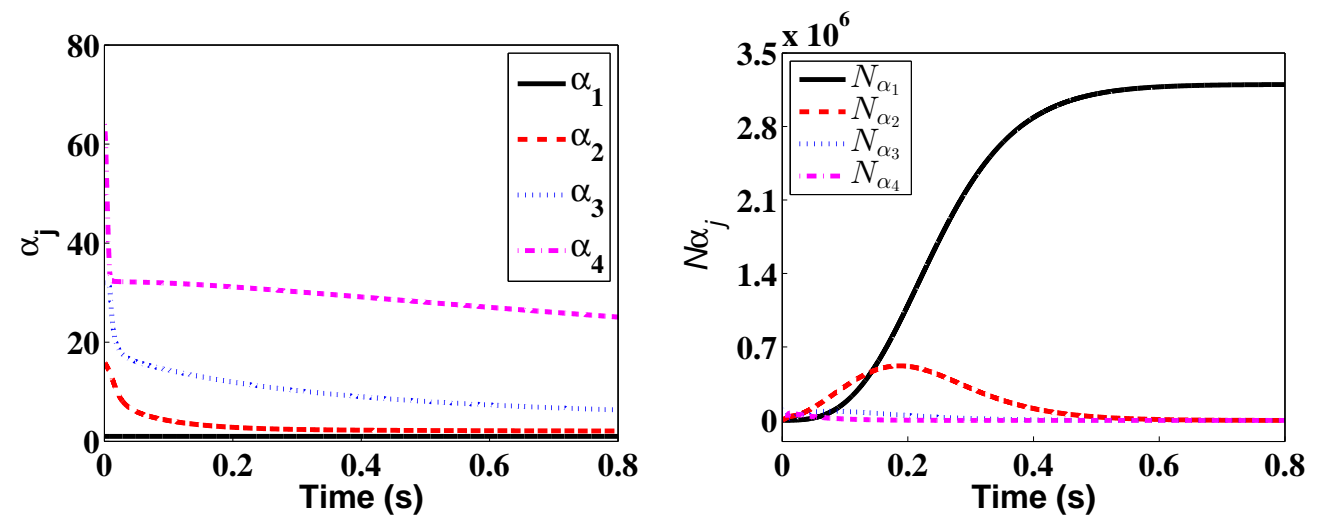

Figure 1: Evolution of the particle mass $\alpha_{j}$ (left panel) and the corresponding number of particles $N_{\alpha_{j}}$ (right panel) obtained using MPM for case 1 .

$$
M_{k, \text { error }}=\frac{\left|\widetilde{M}_{k}-M_{k}\right|}{M_{k}},
$$

where $M_{k}$ is the $k$-th order moment from a high-precision stochastic solution. Figure 2 shows the relative moment errors computed using MPM with $N_{\mathrm{p}}=4$

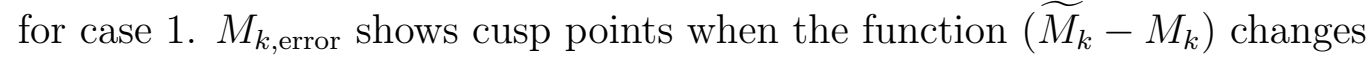
sign which was also observed in Ref. [20] for QMOM. In general, MPM shows very high accuracy. Although the relative errors in the higher-order moments $(k=5,6)$ show an overall increase, the errors at $t=0.8 \mathrm{~s}$ is at most $10^{-4}$. By contrast, the relative errors in the lower-order moments $(k=0,2)$ show an overall decrease. Note that as mass is conserved in MPM the errors in the first-order moment (total particle mass) is 0 .

To investigate the sensitivity of the results to the number of particle masses, $N_{\mathrm{p}}$, moments are computed using MPM with $N_{\mathrm{p}}=3,4$ and 5 and compared with the stochastic solution. Figure 3 shows that for case 1 at least four particle masses (dotted line) are required for there to be no obvious 


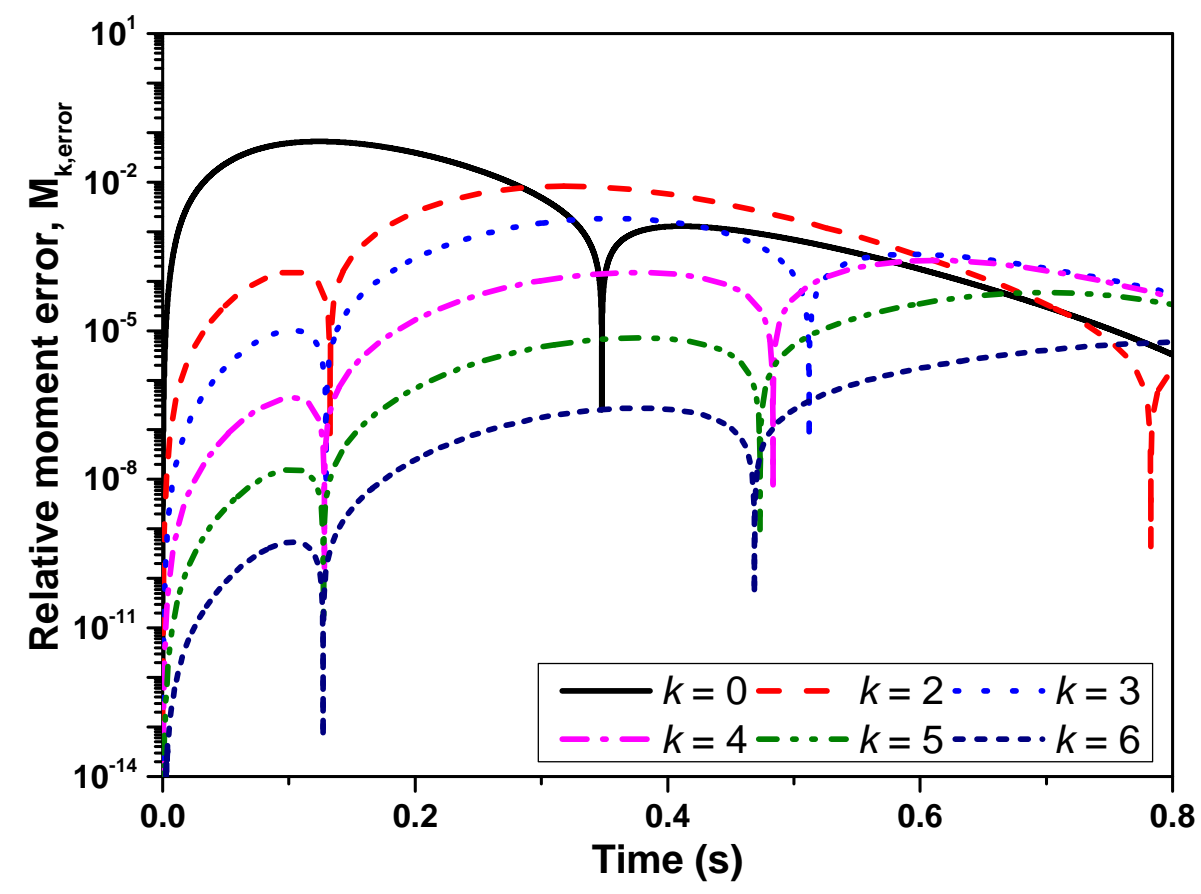

Figure 2: Error in the $k$-th order moment obtained using MPM relative to a highprecision stochastic solution for case 1.

discrepancy in $\widetilde{M}_{0}$. Interestingly, $\widetilde{M}_{0}$ at longer residence times displays little sensitivity to $N_{\mathrm{p}}$. The time-averaged ( $t=0$ to $0.8 \mathrm{~s}$ ) relative moment errors, $M_{k \text {,error }}$, as a function of $N_{\mathrm{p}}$ and $k$ for case 1 are listed in Table 3. As expected, higher accuracy is generally observed when more particle masses are used: there is about an order-of-magnitude decrease in the errors in the lower order moments $(k=0,2,3)$ when $N_{\mathrm{p}}$ is increased from 3 to 5 . However, this is not the case for the higher order moments $(k=4,5,6)$ where there is in fact an increase in errors when $N_{\mathrm{p}}$ is increased from 4 to 5 .

For Case 2 particles undergo erosion where the parent particle mass class is reduced by one and a particle of the smallest mass class is formed. The 


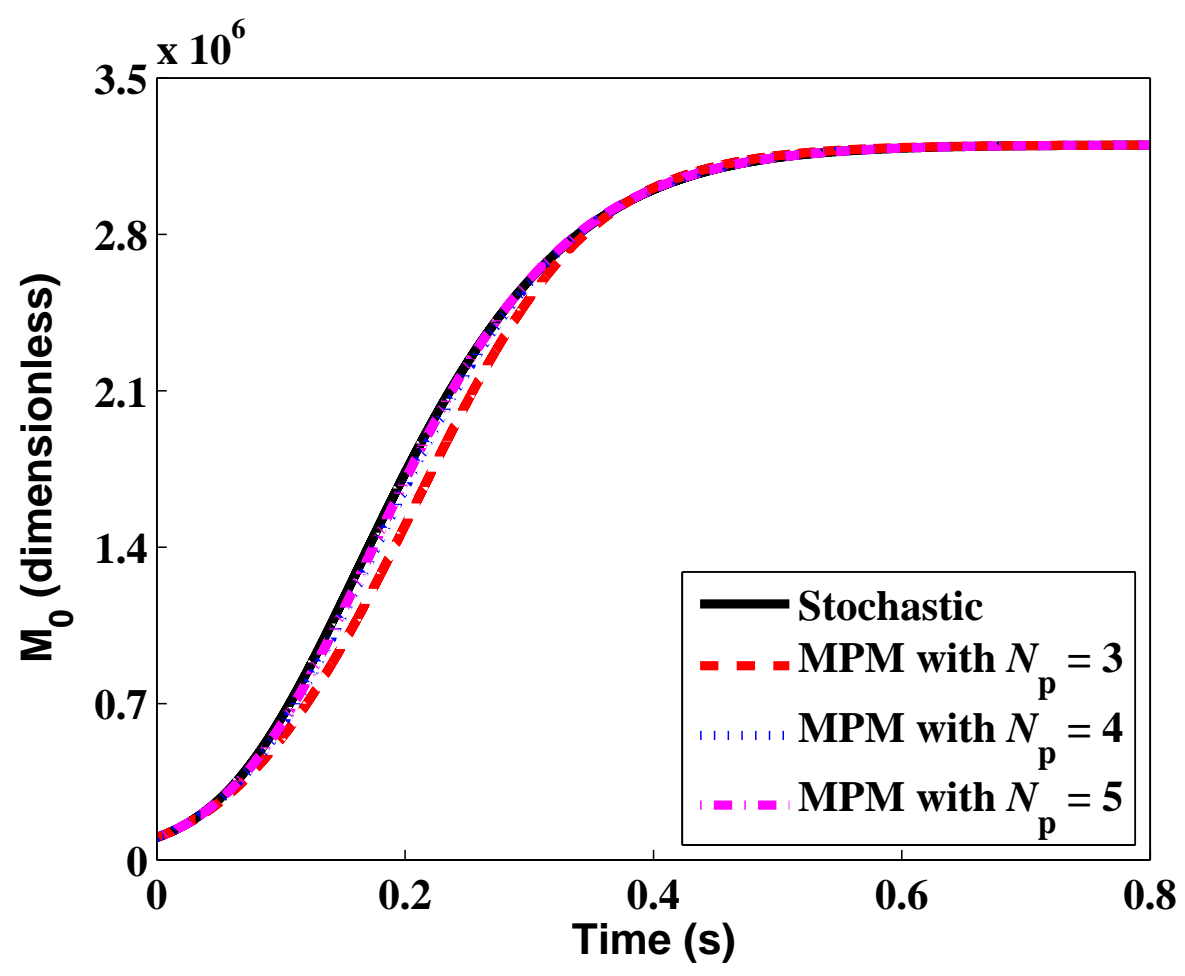

Figure 3: Sensitivity of the zeroth moment $M_{0}$ to the number of particle masses $N_{p}$ obtained using MPM for case 1. The stochastic solution (continuous line) is shown as a point of reference.

rate is controlled by a mass-dependent kernel and a unimodal distribution is supplied as the initial condition. The moment transport equation with the fragmentation moment source term in Eq. (27) is solved. The time evolution of $\alpha_{j}$ and $\widetilde{N}_{\alpha_{j}}$ obtained using MPM is shown in Fig. 4. At $t=0$, the third and fourth particle masses are positioned on either side of the particles at mass class $i=30$. As these particles reduce in mass, $\alpha_{j}(j=2,3,4)$ all move towards the position of the new parent particle class to better represent these particles. This is reflected as an increase in $\alpha_{2}$ (and $\alpha_{3}$ ) and a decrease in $\alpha_{4}$. The evolution of $\widetilde{N}_{\alpha_{j}}$ is similar to that of case 1 . 
Table 3: Average error in the $k$-th order moment obtained using MPM relative to a highprecision stochastic solution for different particle masses $N_{p}$ for case 1.

\begin{tabular}{cccc}
\hline $\mathrm{k}$ & $N_{\mathrm{p}}=3$ & $N_{\mathrm{p}}=4$ & $N_{\mathrm{p}}=5$ \\
\hline 0 & $3.9 \times 10^{-2}$ & $1.3 \times 10^{-2}$ & $8.2 \times 10^{-3}$ \\
1 & 0 & 0 & 0 \\
2 & $8.8 \times 10^{-3}$ & $2.3 \times 10^{-3}$ & $9.7 \times 10^{-4}$ \\
3 & $2.3 \times 10^{-3}$ & $5.2 \times 10^{-4}$ & $2.1 \times 10^{-4}$ \\
4 & $4.0 \times 10^{-4}$ & $9.6 \times 10^{-5}$ & $2.3 \times 10^{-4}$ \\
5 & - & $1.6 \times 10^{-5}$ & $2.8 \times 10^{-4}$ \\
6 & - & $1.2 \times 10^{-6}$ & $3.1 \times 10^{-4}$ \\
7 & - & - & $3.1 \times 10^{-4}$ \\
8 & - & - & $3.2 \times 10^{-4}$ \\
\hline
\end{tabular}
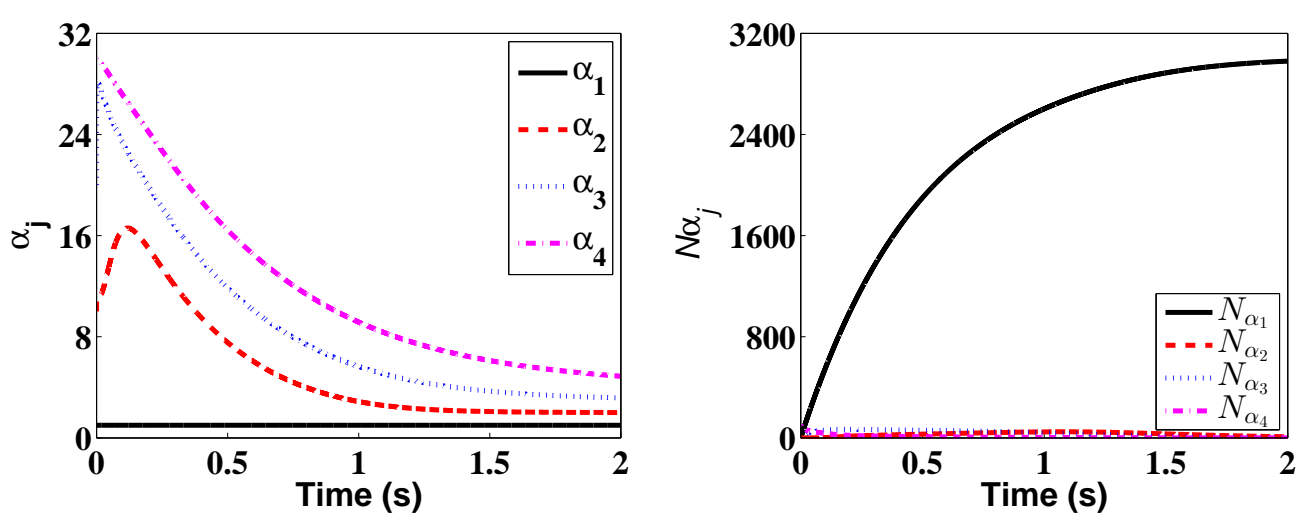

Figure 4: Evolution of the particle mass $\alpha_{j}$ (left panel) and the corresponding number of particles $N_{\alpha_{j}}$ (right panel) computed using MPM for case 2.

Figure 5 shows the sensitivity of $M_{0}$ to the number of particle masses 


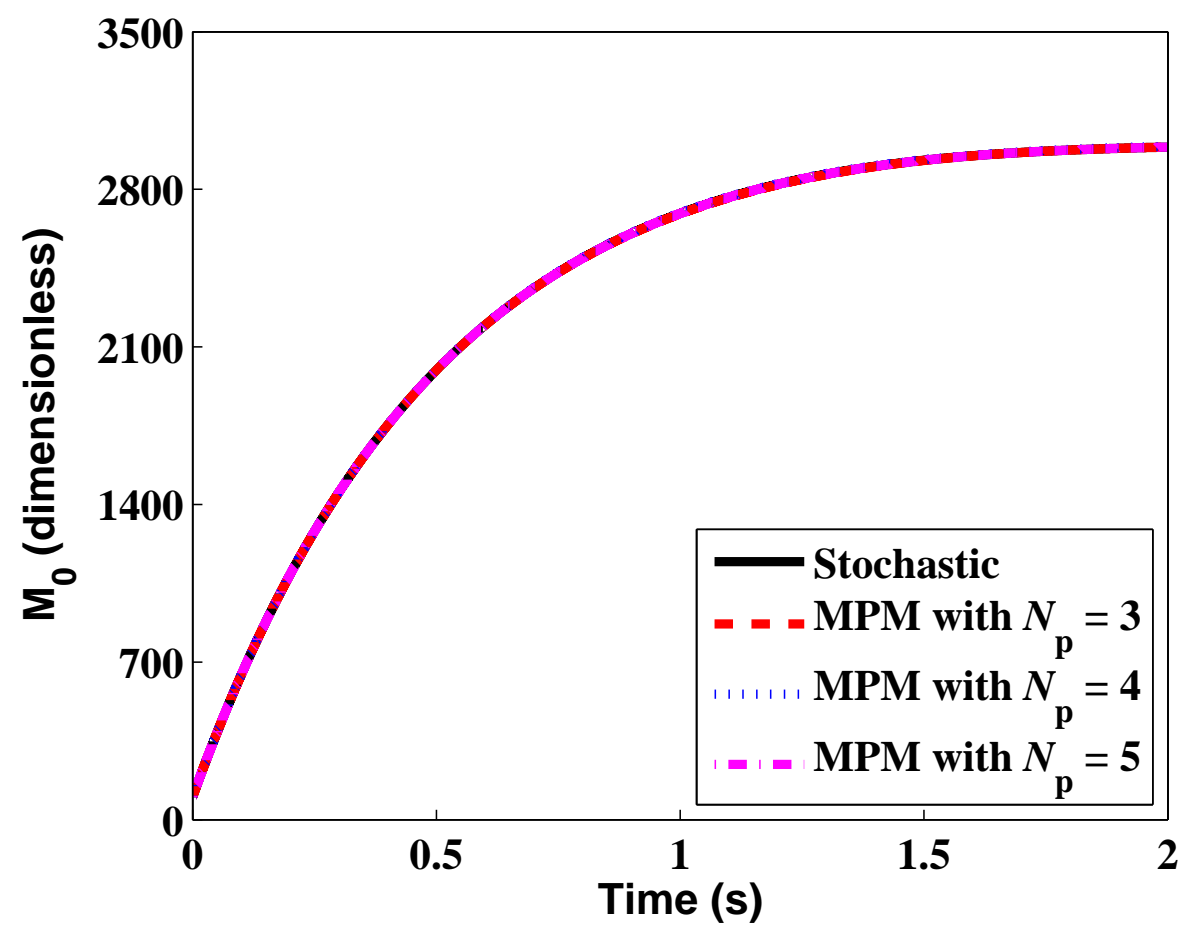

Figure 5: Sensitivity of the zeroth moment $M_{0}$ to the number of particle masses $N_{p}$ obtained using MPM for case 2. The stochastic solution (continuous line) is shown as a point of reference.

difference between MPM and the stochastic method across all particle masses.

This is due to the mass-dependent kernel used where the only source of error in the fragmentation moment source term $F_{k}\left(\widetilde{M}, \widetilde{N}_{\alpha_{1}}\right)$ is in $\widetilde{N}_{\alpha_{1}}$ (see Eqs. (26) and (27) for $k=0$ ) as opposed to both $\widetilde{M}_{0}$ and $\widetilde{N}_{\alpha_{1}}$ for mass-independent kernels (see Eqs. (24) and (25) for $k=0$ ) such as in case 1 . The timeaveraged relative errors $(t=0$ to $2 \mathrm{~s})$ are listed in Table 4 . Overall, the errors are lower than in case 1 but the observations that can be made are similar. Note that each increment in the number of particle masses requires the solution of two extra moments (See Eq. (16)). Smaller tolerances have to 
Table 4: Average error in the $k$-th order moment obtained using MPM relative to a highprecision stochastic solution for different particle masses $N_{p}$ for case 2.

\begin{tabular}{cccc}
\hline $\mathrm{k}$ & $N_{\mathrm{p}}=3$ & $N_{\mathrm{p}}=4$ & $N_{\mathrm{p}}=5$ \\
\hline 0 & $2.7 \times 10^{-4}$ & $1.1 \times 10^{-4}$ & $1.5 \times 10^{-5}$ \\
1 & 0 & 0 & 0 \\
2 & $3.6 \times 10^{-6}$ & $8.4 \times 10^{-8}$ & $5.9 \times 10^{-8}$ \\
3 & $1.9 \times 10^{-6}$ & $8.3 \times 10^{-8}$ & $6.7 \times 10^{-8}$ \\
4 & $1.8 \times 10^{-6}$ & $5.8 \times 10^{-8}$ & $9.3 \times 10^{-8}$ \\
5 & - & $5.4 \times 10^{-8}$ & $9.6 \times 10^{-8}$ \\
6 & - & $5.3 \times 10^{-8}$ & $9.4 \times 10^{-8}$ \\
7 & - & - & $8.8 \times 10^{-8}$ \\
8 & - & - & $8.1 \times 10^{-8}$ \\
\hline
\end{tabular}

be used for the time integration of the set of ODEs and increases the stiffness of the eigenvalue-eigenvector problem solved via the Blumstein and Wheeler algorithm, thus leading to a higher computational cost. For this reason, four particle masses will be used in the rest of this paper.

Case 3 is similar to case 2 except that a mass-dependent kernel is used. The moment transport equation with the fragmentation moment source term in Eq. (26) is solved. We now compare MPM to other moment methods: HMOM and QMOM with four nodes. Figure 6 shows a comparison of $M_{0}$ between MPM, HMOM and QMOM with the stochastic solution as a reference. There is an excellent agreement between MPM and the stochastic method apart from a slight underprediction at intermediate times. Both HMOM and QMOM overestimate $M_{0}$ but the performance by HMOM is worse. It 


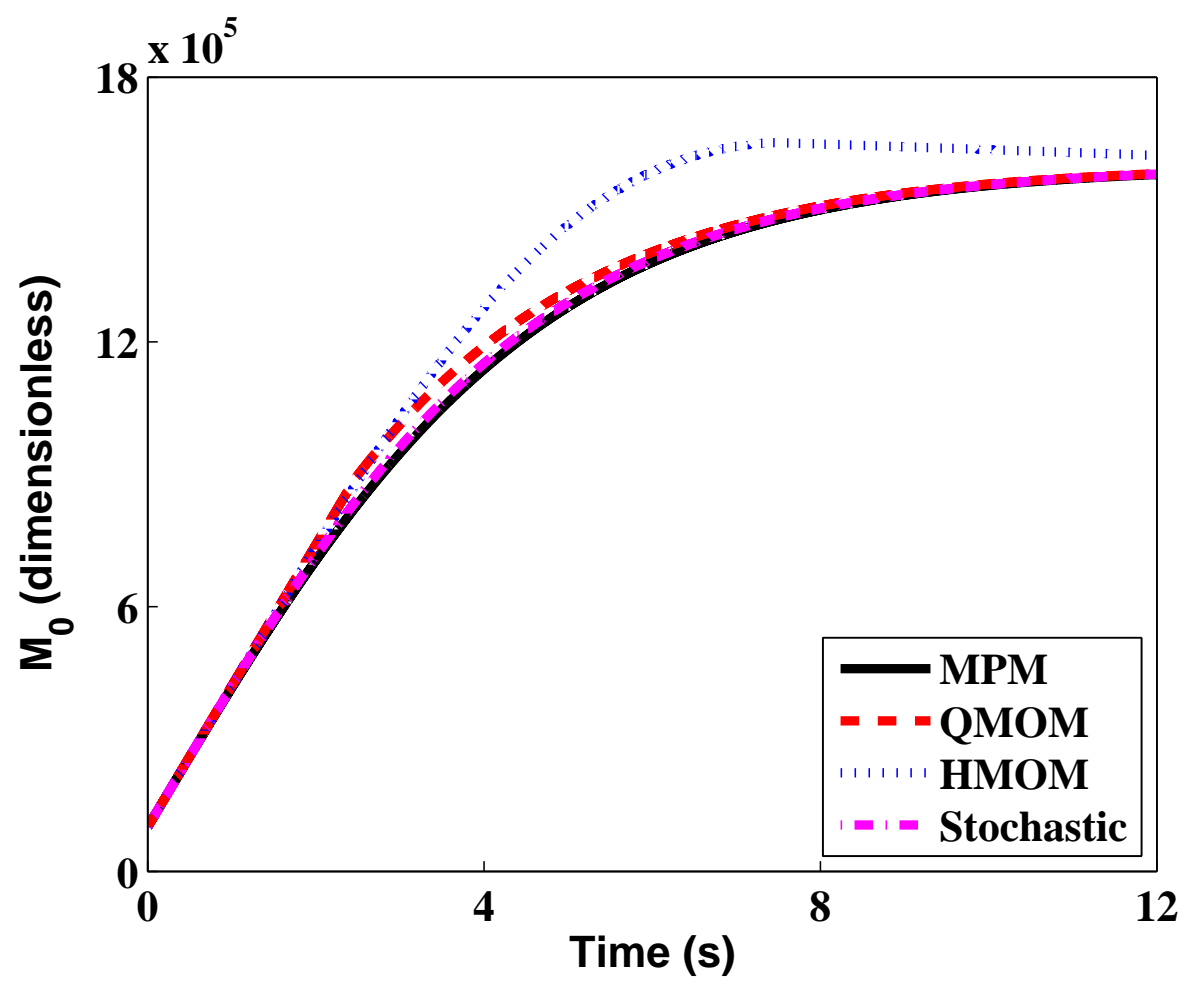

Figure 6: Comparison of the zeroth moment $M_{0}$ between MPM (four particle masses), QMOM (four nodes), HMOM and the stochastic solution for case 3.

was initially puzzling but it became clear to us that in HMOM particles are represented as either small or large particles which is a coarser assumption than the four particles masses or nodes used in MPM and QMOM, respectively. Second, it is assumed that the rate at which the smallest particles are formed is proportional to the overall fragmentation rate [7]. However, there exist situations where particles fragment and the smallest particles are not formed, for example, in symmetric fragmentation. Although QMOM incurs some errors, when particles are small enough, it implicitly tracks the number of the smallest particles which keeps its accuracy high. The results for case 4 

(see Fig. 7).

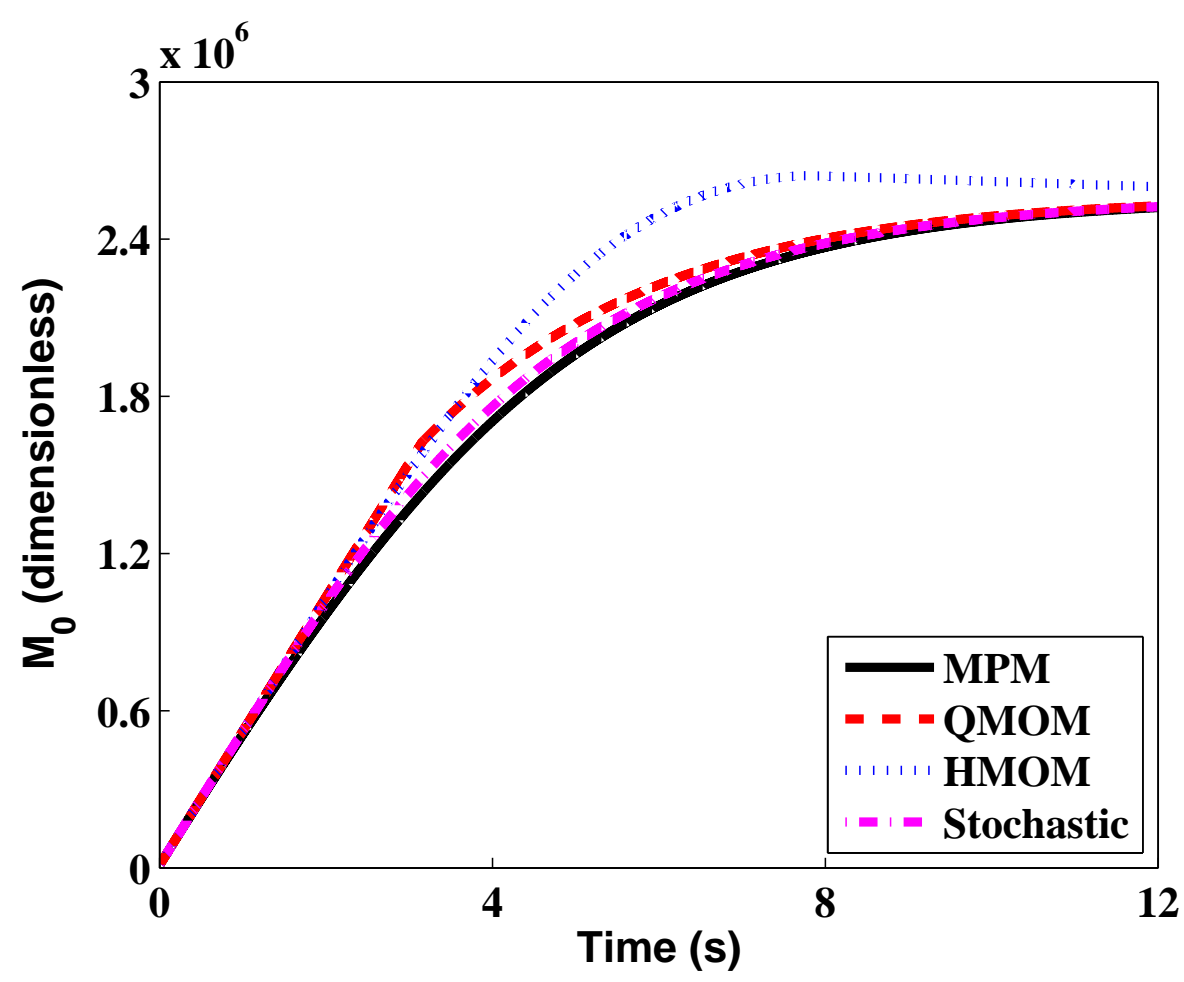

Figure 7: Comparison of the zeroth moment $M_{0}$ between MPM (four particle masses), QMOM (four nodes), HMOM and the stochastic solution for case 4.

333

where a unimodal distribution is supplied as the initial condition is similar

For case 5, particles undergo erosion with a constant kernel and the moment transport equation with the fragmentation source term in Eq. (25) is solved. Unlike case 2 where there are only particles at mass class $i=30$ at $t=0 \mathrm{~s}$, the parabolic distribution for this case has particles in the smallest mass class. Therefore, the ability to accurately track the number of the smallest particles is particularly important. Both HMOM and QMOM are not able to even capture the steady-state $M_{0}$ at $t=20 \mathrm{~s}$ as shown in Fig. 8. 


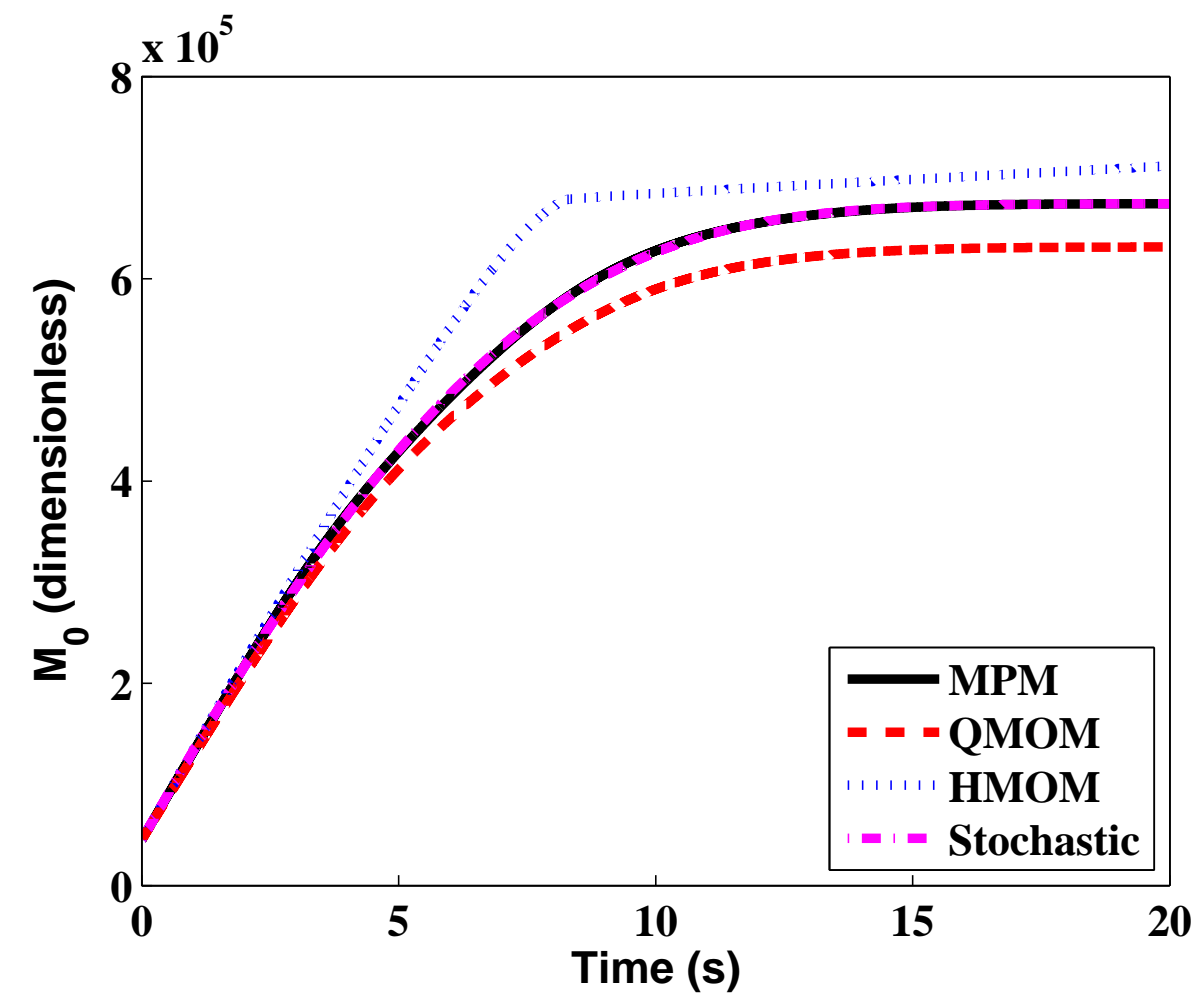

Figure 8: Comparison of the zeroth moment $M_{0}$ between MPM (four particle masses), QMOM (four nodes), HMOM and the stochastic solution for case 5.

For cases 6 and 7, particles undergo erosion and a log-normal distribution is supplied as the initial condition. A constant fragmentation kernel is used in case 6 while a mass-dependent fragmentation kernel is used in case 7 . $M_{0}$ computed using the different methods for cases 6 and 7 are shown in Figs. 9 and 10, respectively. The results for case 6 is similar to case 5 where HMOM overpredicts and QMOM underpredicts $M_{0}$. When a massdependent fragmentation kernel is used in Case 7, the agreement is much improved. As highlighted before, one reason for the improved performance is that when the mass-dependent kernel is used, the source term for the zeroth- 


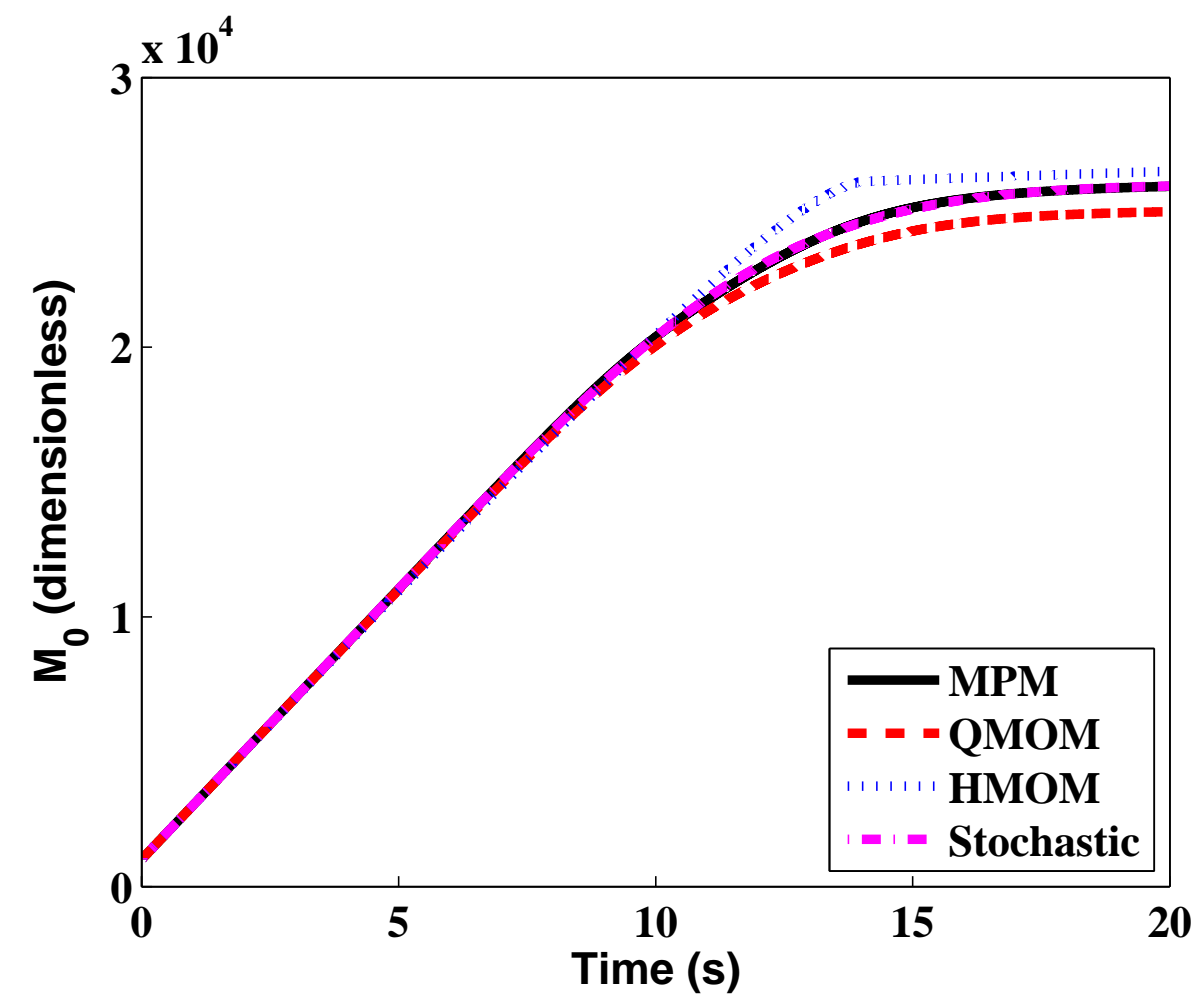

Figure 9: Comparison of the zeroth moment $M_{0}$ between MPM (four particle masses), QMOM (four nodes), HMOM and the stochastic solution for case 6.

order moment is governed by the total particle mass which is insensitive to the number of the smallest particles, thus decreasing the errors in computing the moments. In both cases, MPM exhibits the highest accuracy regardless of the fragmentation kernel used.

Based on the above results, the following observations can be made: MPM is the most accurate amongst the different method of moments studied for the pure fragmentation process. Across all of these test cases, the agreement between $M_{0}$ obtained using MPM and the stochastic method is excellent. The source term developed in HMOM tends to overestimate the formation of the 


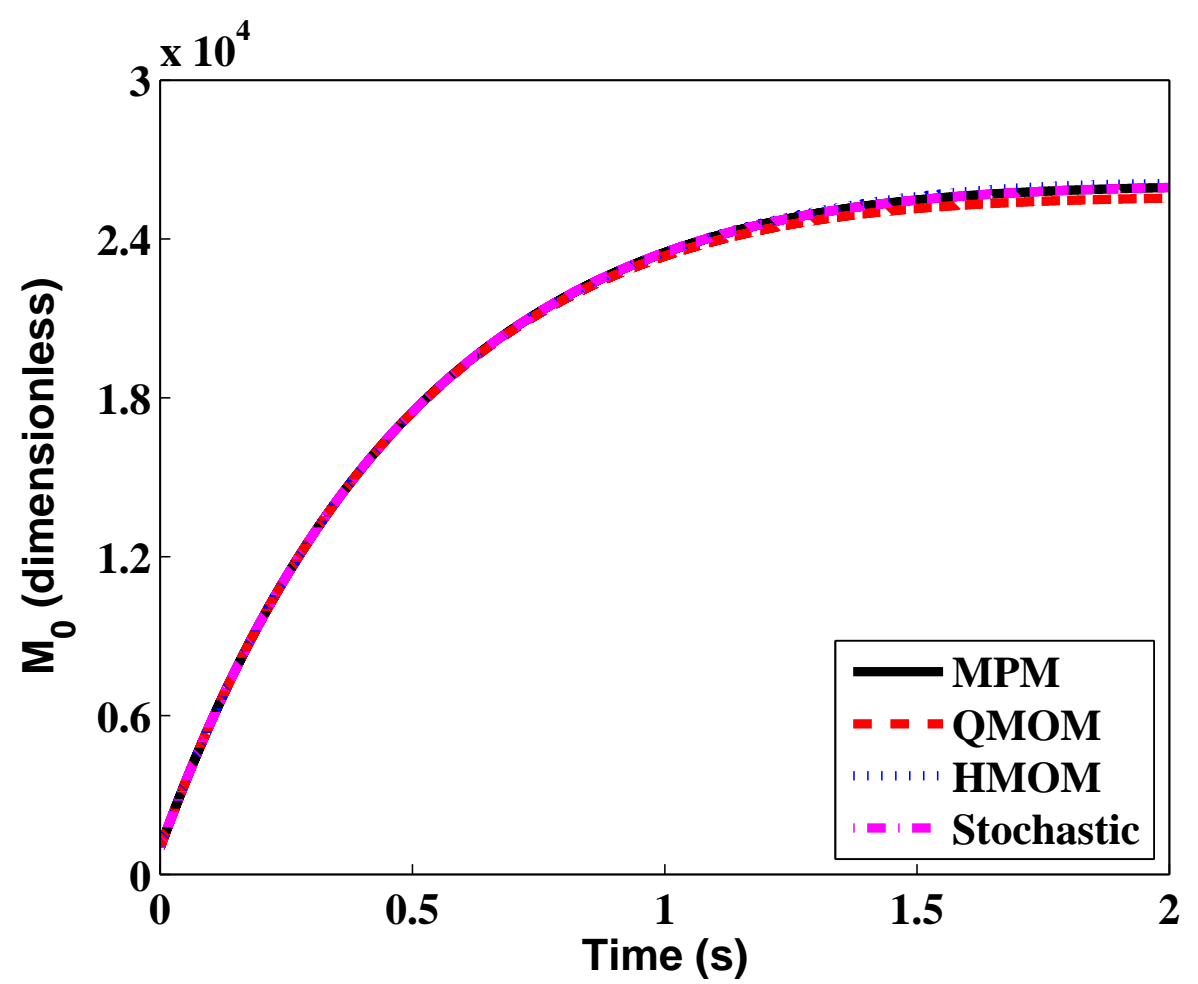

Figure 10: Comparison of the zeroth moment $M_{0}$ between MPM (four particle masses), QMOM (four nodes), HMOM and the stochastic solution for case $\%$

smallest particles. Because QMOM does not explicitly track the number of the smallest particles, the performance of QMOM is worse for erosion than for symmetric fragmentation.

\subsection{Simultaneous coagulation and fragmentation}

In this section, the performance of MPM is tested for simultaneous coagulation and fragmentation processes. Depending on the coagulation and fragmentation kernels used, the PSD will evolve differently and result in different total particle numbers at steady state. Four cases are developed to investigate the competition between these two processes as shown in Table 5. 
The fragmentation kernel is systematically varied while the coagulation ker-

Table 5: Cases used for the comparison of simultaneous coagulation and fragmentation.

\begin{tabular}{ll}
\hline Case & $K_{\mathrm{Fg}}(i)$ \\
\hline 9 & $\begin{cases}0 & i=1 \\
0.02 & i>1 \\
0 & i=1 \\
200 & i>1 \\
0 & i=1 \\
0.02 i & i>1 \\
0 & i=1 \\
200 i & i>1\end{cases}$ \\
\hline
\end{tabular}

Note: $K_{\mathrm{Cg}}=0.02 \mathrm{~s}^{-1}, P(i \mid j)=$ erosion, $N_{30}(t=0)=100$.

nel is left unchanged. For all of these cases, fragmentation takes the form of erosion and the unimodal distribution in case 2 is supplied as the initial condition.

For case 8 , the coagulation and fragmentation kernels are identical. $M_{0}$ computed using the different methods are shown in the left panel of Fig. 11. The process is dominated by coagulation as shown by the decrease in $M_{0}$. Therefore, very few particles accumulate in the first particle mass class as these particles tend to collide with each other to form particles of larger mass. Since constant kernels are used, no closure problem is present in the coagulation moment equation and all the methods generate almost the same results as the stochastic method. Also shown in Fig. 11 (right panel) are the corresponding results for case 9 where the fragmentation kernel is four orders- 

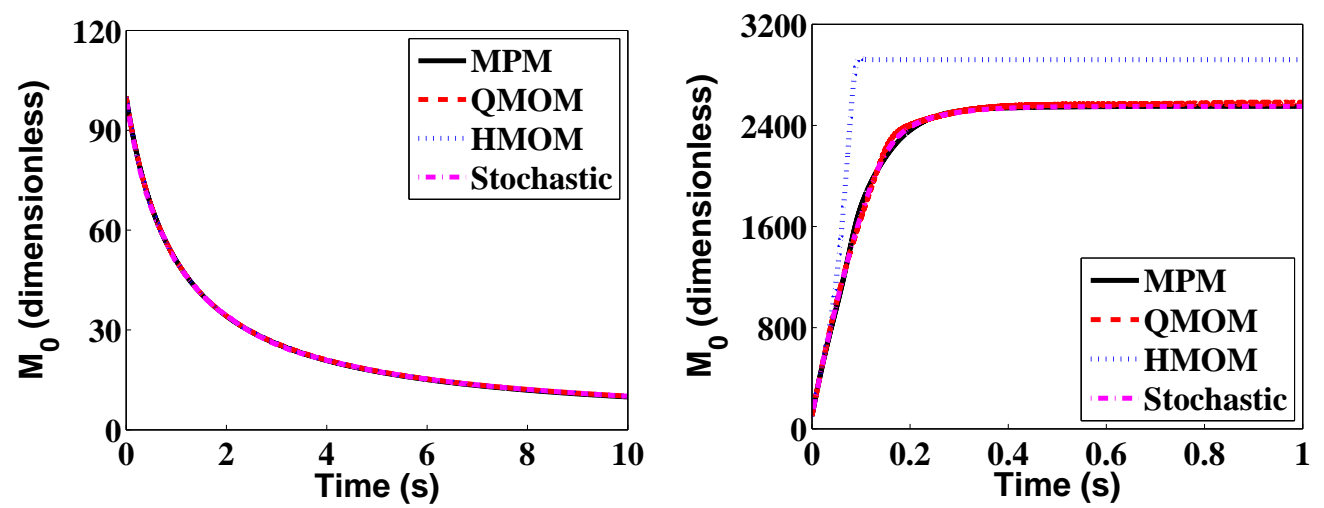

Figure 11: Comparison of the zeroth moment $M_{0}$ between MPM (four particle masses), QMOM (four nodes), HMOM and the stochastic method for case 8 (left panel) and case 9 (right panel).

of-magnitude larger than the coagulation kernel. The process is dominated by fragmentation and the accumulation of the smallest particles plays an important role: HMOM overestimates the formation of the smallest particles, thus overestimating $M_{0}$; MPM shows the highest accuracy while slight discrepancy is observed between the QMOM and stochastic solutions. Cases 10 and 11 are similar to cases 8 and 9 except that mass-dependent fragmentation kernels are used. Similar conclusions can be drawn from Fig. 12.

\subsection{Combined processes}

In this section, MPM is tested against QMOM, HMOM and the stochastic method for the combined processes of inception, growth, coagulation, shrinkage and fragmentation. The specifics of the two test cases are shown in Table 6. The total particle number and mass of particles computed using the different methods for cases 12 and 13 are shown in Figs. 13 and 14, respectively. It can be seen that MPM exhibits a very high accuracy that was 

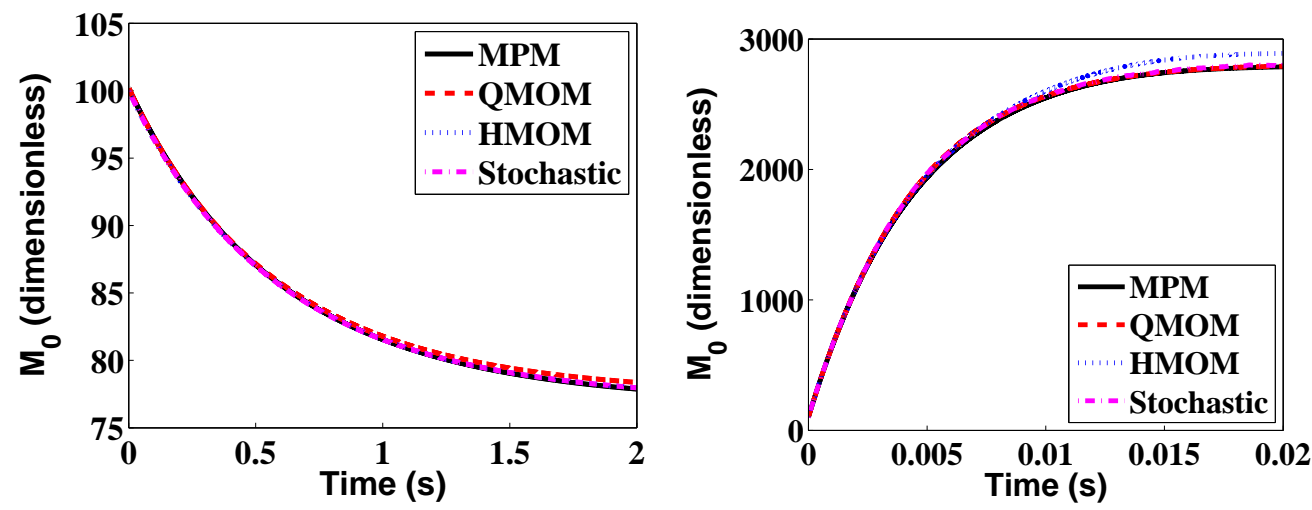

Figure 12: Comparison of the zeroth moment $M_{0}$ between MPM (four particle masses), QMOM (four nodes), HMOM and the stochastic method for case 10 (left panel) and case 11 (right panel).

Table 6: Cases used for the comparison of combined processes.

\begin{tabular}{lll} 
Case & $K_{\mathrm{Fg}}(i)$ & $N_{i}(t=0)$ \\
12 & $\left\{\begin{array}{lll}0 & i=1 & 100 \exp \left(-(\log (i)-\log (25))^{2} / 0.05\right), \\
2 \times 10^{-5} i & i>1 & i=1, \ldots, 100 \\
0 & i=1 \\
2 \times 10^{-5} & i>1\end{array}\right.$ \\
\hline
\end{tabular}

Note: $I_{m_{1}}=100 \mathrm{~s}^{-1}, K_{\mathrm{G}}=20 \mathrm{~s}^{-1}, K_{\mathrm{Cg}}=2 \times 10^{-5} \mathrm{~s}^{-1}, K_{\mathrm{Sk}}=30 \mathrm{~s}^{-1}$ and $P(i \mid j)=$ erosion.

also observed for pure fragmentation and simultaneous coagulation and fragmentation. $M_{0}$ decreases mainly due to the shrinkage of particles-rather than coagulation - as evidenced by the corresponding decrease in $M_{1}$. The shrinkage process leads to a zeroth order moment equation containing a term corresponding to the loss of particles of the smallest size $[12,39]$. In order to 

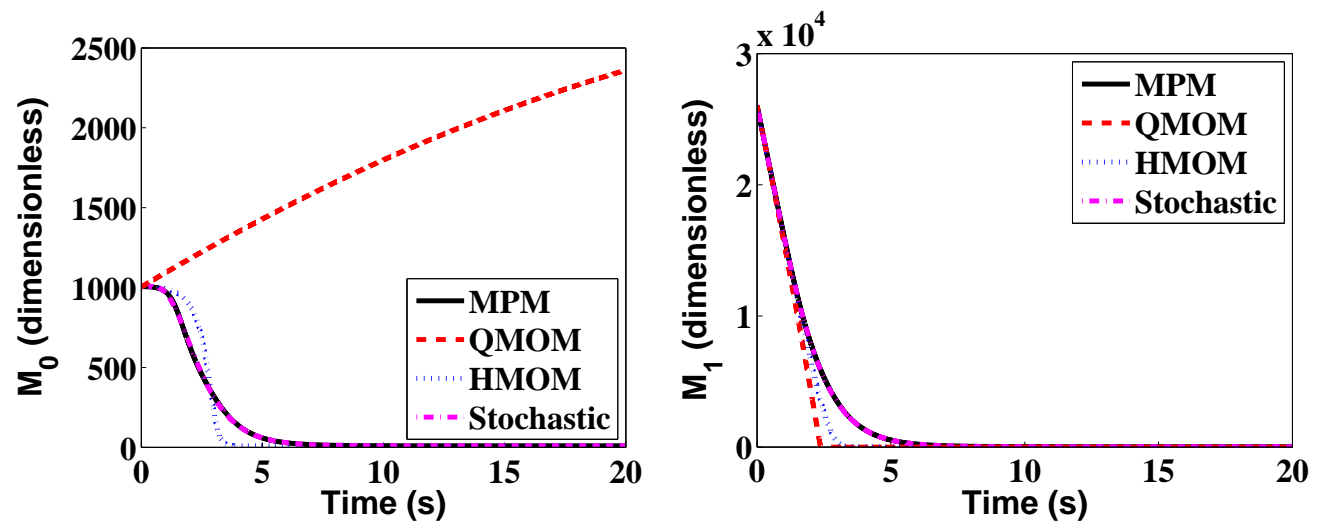

Figure 13: Comparison of the zeroth order moment $M_{0}$ (left panel) and the first order moment $M_{1}$ (right panel) between MPM (four particle masses), QMOM (four nodes), HMOM and the stochastic method for case 12.
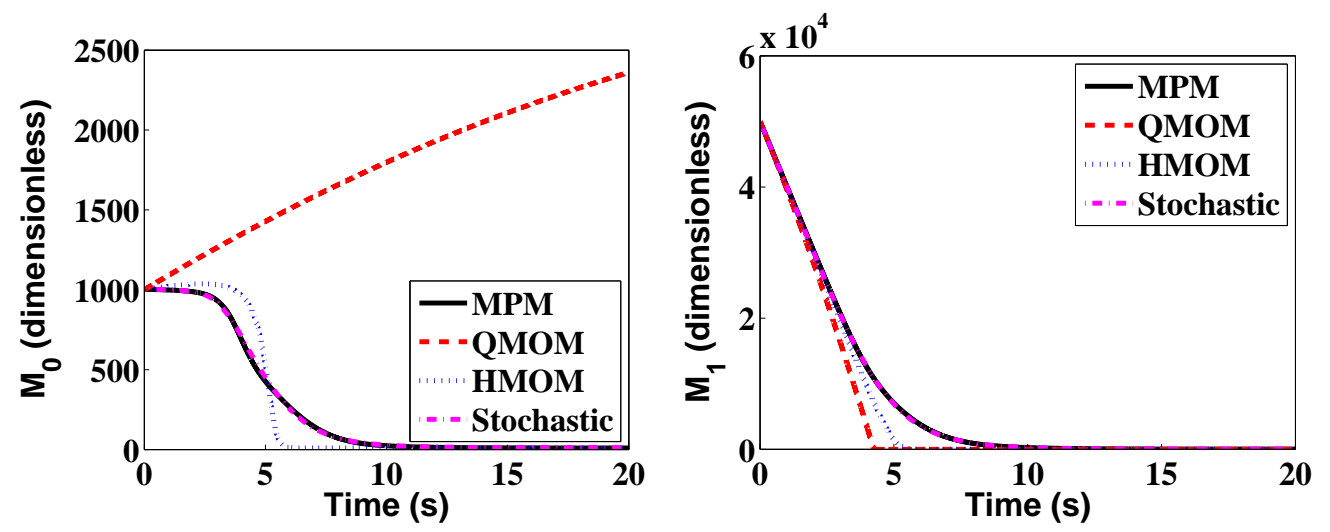

Figure 14: Comparison of the zeroth order moment $M_{0}$ (left panel) and the first order moment $M_{1}$ (right panel) between MPM (four particle masses), QMOM (four nodes), HMOM and the stochastic method for case 13.

400

evaluate this term, the value of the PSD at the smallest internal coordinate is required which is not available in QMOM. As expected, Figs. 13 and 14 show that QMOM fails to predict the evolution of $M_{0}$ and therefore $M_{1}$. Although HMOM is able to predict the consumption of particles, it shows a significant 
404 discrepancy compared with the stochastic solution. 


\section{Conclusion}

In this paper, the moment projection method (MPM) was extended to include the fragmentation process. MPM was tested against cases involving (1) pure fragmentation, (2) simultaneous coagulation and fragmentation, and (3) combined processes of inception, growth, coagulation, shrinkage and fragmentation with different fragmentation kernels, fragment distribution functions and initial conditions. The numerical results were compared against the hybrid method of moments (HMOM) and the quadrature method of moments (QMOM) with four nodes and a high-precision stochastic solution calculated using the direct simulation algorithm (DSA).

By fixing the first particle mass $\alpha_{1}$ to be equal to the smallest particle mass $m_{1}$, the evolution of the smallest particles could be tracked in MPM with a high accuracy. The accuracy was shown to generally improve with the number of particle masses, $N_{\mathrm{p}}$, with $N_{\mathrm{p}}=4$ being the best compromise between accuracy and computational efficiency. In all the test cases considered in this work, MPM is capable of accurately predicting the time evolution of the moments while the agreement with HMOM and QMOM tend to be less good when fragmentation dominates. Future work includes application of MPM to real particle processes such as soot formation in flames. It remains to be seen how effective is MPM for more complicated PBEs with additive kernels and/or free-molecular Brownian kernel.

\section{Acknowledgement}

This research is supported by the National Research Foundation, Prime Minister's Office, Singapore under its CREATE programme. 


\section{Nomenclature}

Upper-case Roman

D Eigenvectors of matrix $\mathbf{P}$

$F \quad$ Source term due to fragmentation

$G$ Source term due to coagulation

$I_{m_{1}}$ Inception rate of particles of the smallest mass $m_{1}$

$K_{\mathrm{Cg}}$ Coagulation kernel

$K_{\mathrm{Fg}} \quad$ Fragmentation kernel

$K_{\mathrm{G}} \quad$ Growth kernel

$K_{\mathrm{Sk}} \quad$ Shrinkage kernel

$430 \quad M \quad$ Moment

$N$ Number

P Symmetric tridiagonal matrix as a function of recursion coefficients $a$ and $b$

$P \quad$ Fragment distribution function

$R$ Source term due to inception

$S$ Source term due to shrinkage

$\mathbf{V}$ Eigenvalues of matrix $\mathbf{P}$

$W \quad$ Source term due to growth

Z Matrix with components $Z$ which are a function of the moments $M$ 


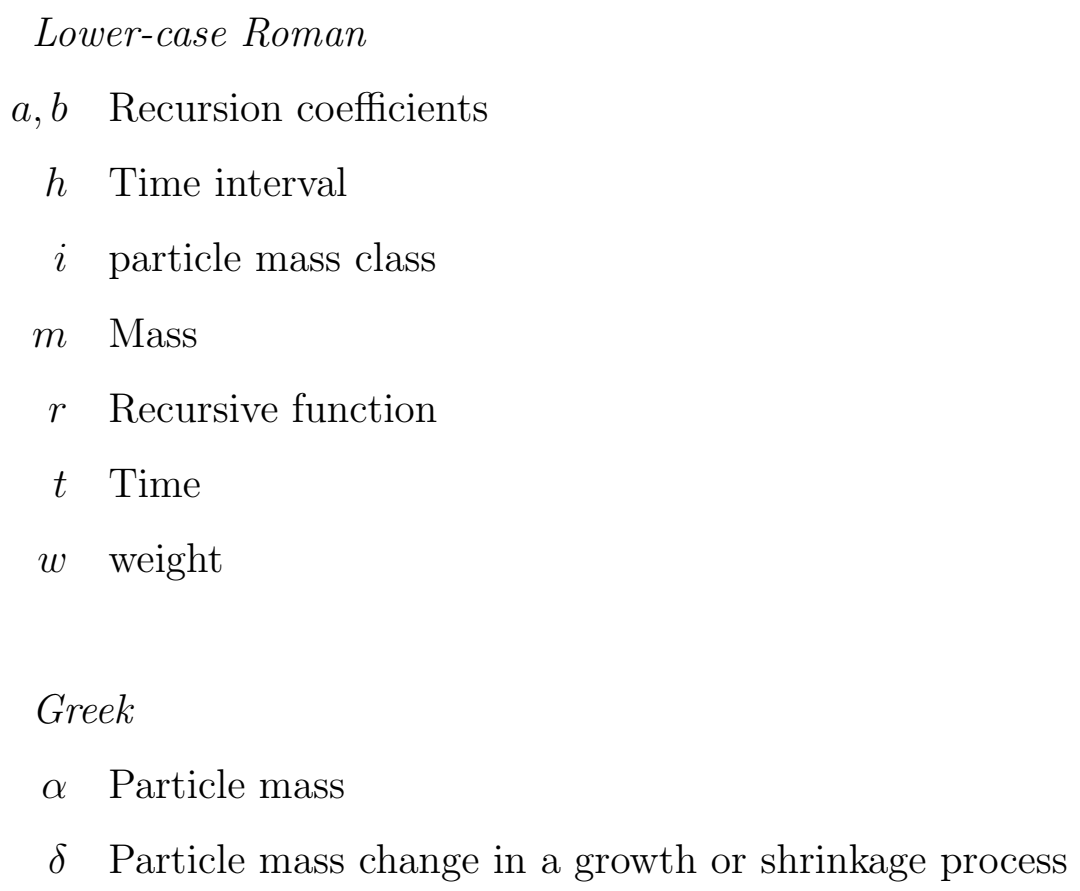


$\widetilde{x} \quad$ Approximation of $x$

$\widehat{b}$ Integral of fragmentation distribution function

\section{Abbreviations}

DQMOM Direct quadrature method of moments

DSA Direct simulation algorithm

EQMOM Extended quadrature method of moments

FCMOM Finite-size domain complete set of trial functionss method of mo-

432 ments

HMOM Hybrid method of moments

MOM Method of moments

MOMIC Method of moments with interpolative closure

MPM Moment projection method

ODE Ordinary differential equation

PBE Population balance equation

PSD Particle size distribution

QMOM Quadrature method of moments

\section{Appendix A. Blumstein-Wheeler algorithm}

This algorithm is used to determine the particle masses and the numbers used to approximate the PSD from the empirical moments. The algorithm is implemented in Matlab and makes use of the eig function to determine the eigenvalues and eigenvectors. 
Algorithm 2: Blumstein-Wheeler algorithm.

Input: The empirical moments $\widetilde{M}_{k}$ for $k=0,1, \ldots, 2 N_{\mathrm{p}}-2$.

Output: The particle masses $\alpha_{j}$ and the corresponding number of particles $\widetilde{N}_{\alpha_{j}}$ for

$$
j=1,2, \ldots, N_{\mathrm{p}} .
$$

Create a $N_{\mathrm{p}} \times 2 N_{\mathrm{p}}$ matrix $\mathbf{Z}$ with zeros in all elements.

Determine the elements of the first row of matrix $\mathbf{Z}: Z_{1, l}=\widetilde{M}_{l-1}$ for $l=1, \ldots, 2 N_{\mathrm{p}}-1$.

For $a_{1}=\widetilde{M}_{1} / \widetilde{M}_{0}$ and $b_{1}=0$, determine the recursion coefficients $a_{k}$ and $b_{k}$ :

for $k=2$ to $N_{p}$ do

for $l=k$ to $2 N_{p}-1$ do

The elements of $\mathbf{Z}$ must satisfy the following recursion relation:

$$
\begin{array}{r}
Z_{k, l}=Z_{k-1, l+1}-a_{k-1} Z_{k-1, l}-b_{k-1} Z_{k-1, l} ; \\
a_{k}=\frac{Z_{k, k+1}}{Z_{k, k}}-\frac{Z_{k-1, k}}{Z_{k-1, k-1}} ; \quad b_{k}=\frac{Z_{k, k}}{Z_{k-1, k-1}} .
\end{array}
$$

For $r_{1}=1 /\left(m_{1}-a_{1}\right)$ where $m_{1}$ is the smallest particle mass, determine the recursion function:

$$
r_{k}=1 /\left(m_{1}-a_{k}-b_{k} r_{k-1}\right), \quad k=2, \ldots, N_{\mathrm{p}}-1 .
$$

As we fix the smallest particle mass, replace $a_{N_{\mathrm{p}}}$ with:

$$
a_{N_{\mathrm{p}}}=m_{1}-b_{N_{\mathrm{p}}} r_{N_{\mathrm{p}}-1}
$$

Construct a symmetric tridiagonal matrix $\mathbf{P}$ with $a_{k}$ as the diagonal and the square roots of $b_{k}$ as the co-diagonal:

$$
\mathbf{P}=\left[\begin{array}{ccccc}
a_{1} & -\sqrt{b_{2}} & 0 & \cdots & 0 \\
-\sqrt{b_{2}} & a_{2} & -\sqrt{b_{3}} & \ldots & 0 \\
0 & -\sqrt{b_{3}} & a_{3} & \cdots & 0 \\
\vdots & \vdots & \vdots & \ddots & \vdots \\
0 & 0 & 0 & \cdots & a_{\mathrm{N}_{p}}
\end{array}\right]
$$

Solve for the eigenvalues $\mathbf{V}$ and eigenvectors $\mathbf{D}$ of matrix $\mathbf{P}$ :

$$
[\mathbf{V}, \mathbf{D}]=\operatorname{eig}(\mathbf{P})
$$

Solve for $\alpha_{j}$ and $\widetilde{N}_{\alpha_{j}}$ :

$$
\alpha_{j}=\mathbf{V}(j, j), \quad \widetilde{N}_{\alpha_{j}}=\widetilde{M}_{0} \mathbf{D}(1, j)^{2}
$$




\section{Appendix B. Hybrid method of moments}

The hybrid method of moments (HMOM) was originally developed for bivariate population balance equations (PBEs) based on particle volume and surface area $[6,7]$. Here we revise the method so that it is applicable to monovariate PBEs. Below is a brief description of HMOM based on particle mass for symmetric fragmentation with a constant kernel.

Following the idea in Ref. [6], the particles are discretised into two modes: particles of the smallest mass class $i_{0}$ and particles of the large mass class $i_{\mathrm{L}}$. The moments can then be represented as:

$$
M_{k}=N_{i_{0}} i_{0}^{k}+N_{i_{\mathrm{L}}} i_{\mathrm{L}}^{k}
$$

where $N_{i_{0}}$ and $N_{i_{\mathrm{L}}}$ are the number of particles of mass $i_{0}$ and $i_{\mathrm{L}}$, respectively. The fragmentation moment source term for symmetric fragmentation with a constant kernel (Eq. (24)) can then be written as:

$$
\frac{\mathrm{d} M_{k}}{\mathrm{~d} t}= \begin{cases}K_{\mathrm{Fg}} N_{i_{\mathrm{L}}}, & k=0, \\ 0, & k=1, \\ \left(2^{1-k}-1\right) K_{\mathrm{Fg}} i_{\mathrm{L}}^{k} N_{i_{\mathrm{L}}}, & k>1 .\end{cases}
$$

The source term for $N_{i_{0}}$ is given by the negative infinity order moments:

$$
\frac{\mathrm{d} N_{i_{0}}}{\mathrm{~d} t}=\lim _{k \rightarrow-\infty} \frac{\mathrm{d} M_{k} / \mathrm{d} t}{i_{0}^{k}} .
$$

Applying Eq. (B.3) to Eq. (6) for symmetric fragmentation, we obtain:

$$
\frac{\mathrm{d} N_{i_{0}}}{\mathrm{~d} t}=2 K_{\mathrm{Fg}} N_{2 i_{0}}
$$


The only unknown term $N_{2 i_{0}}$ corresponds to the intermodal transfer of particles from the second mode to the first during the fragmentation process. To close this term, in Ref. [6] it is assumed that the rate of transfer is proportional to the overall fragmentation rate with a coefficient equal to the mass ratio between the two modes $i_{0} / i_{L}$. As a result, Eq. (B.4) can be transformed into:

$$
\frac{\mathrm{d} N_{i_{0}}}{\mathrm{~d} t}=\frac{2 i_{0}^{2}}{i_{\mathrm{L}}^{2}} K_{\mathrm{Fg}} N_{i_{\mathrm{L}}} .
$$

assuming the remaining two quantities in Eq. (B.1) are obtained by inverting the system with two known moments:

$$
N_{i_{\mathrm{L}}}=M_{0}-N_{i_{0}},
$$

and

$$
i_{\mathrm{L}}=\frac{M_{1}-N_{i_{0}} i_{0}}{N_{i_{\mathrm{L}}}} .
$$

Algorithm 3 describes the numerical procedure of HMOM for symmetric fragmentation with a constant kernel. HMOM for other processes (inception, growth, shrinkage, coagulation, symmetric fragmentation with a massdependent kernel, erosion fragmentation with a constant or mass-dependent kernel) can be obtained in a similar way. The details are not given here for simplicity. 


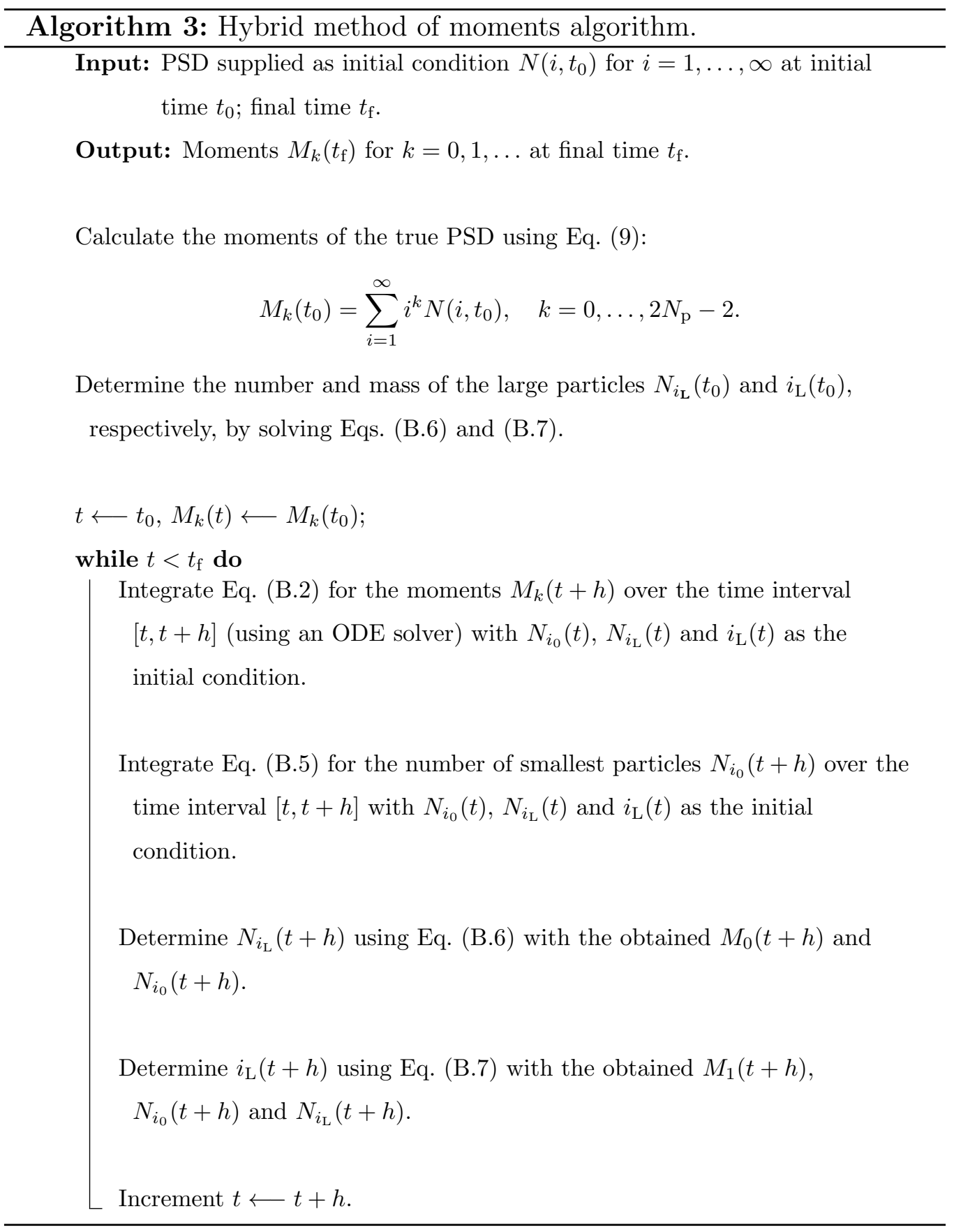


481

$$
\widehat{b}\left(i_{\alpha}\right)=\sum_{i=1}^{i_{\alpha}} i^{k} P\left(i \mid i_{\alpha}\right) .
$$
480 Eq. (C.2) to Eq. (C.1) leads to

\section{Appendix C. Qudrature method of moments}

The quadrature method of moments (QMOM) used in this work is similar to the one in Ref. [20]. This method was originally derived from continuous PSD approaches. Here we give a simple description about the way QMOM is used for fragmentation processes with a discrete-mass distribution.

In order to apply the QMOM, the fragmentation equation must first be transformed into moment equation which is the same as Eq. (15):

$$
\frac{\mathrm{d} M_{k}}{\mathrm{~d} t}=\sum_{j=1}^{\infty} \sum_{i=1}^{j} K_{\mathrm{Fg}}(j) i^{k} P(i \mid j) N_{j}-\sum_{i=1}^{\infty} K_{\mathrm{Fg}}(i) i^{k} N_{i} .
$$

477 where $N$ is the number of quadrature nodes. $i_{\alpha}$ and $w_{\alpha}$ are respectively the 478 quadrature abscissas and weights and their values can be determined using a 479 product-different (PD) algorithm from lower-order moments [53]. Applying

$$
\frac{\mathrm{d} M_{k}}{\mathrm{~d} t}=\sum_{\alpha=1}^{N} K_{\mathrm{Fg}}\left(i_{\alpha}\right) w_{\alpha} \widehat{b}\left(i_{\alpha}\right)-\sum_{\alpha=1}^{N} i_{\alpha}^{k} K_{\mathrm{Fg}}\left(i_{\alpha}\right) w_{\alpha}
$$

For symmetric fragmentation 


$$
\widehat{b}\left(i_{\alpha}\right)=2^{1-k} i_{\alpha}^{k},
$$

483 and for erosion

$$
\widehat{b}\left(i_{\alpha}\right)=1^{k}+\left(i_{\alpha}-1\right)^{k} .
$$

${ }_{484}$ Note that $K_{\mathrm{Fg}}\left(i_{\alpha}=1\right)=0$ since the smallest particles cannot fragment.

485 Algorithm 4 describes the numerical procedure of QMOM for fragmenta486 tion process. QMOM for other processes can be obtained in a similar way. ${ }_{487}$ The details are not given here for simplicity. 


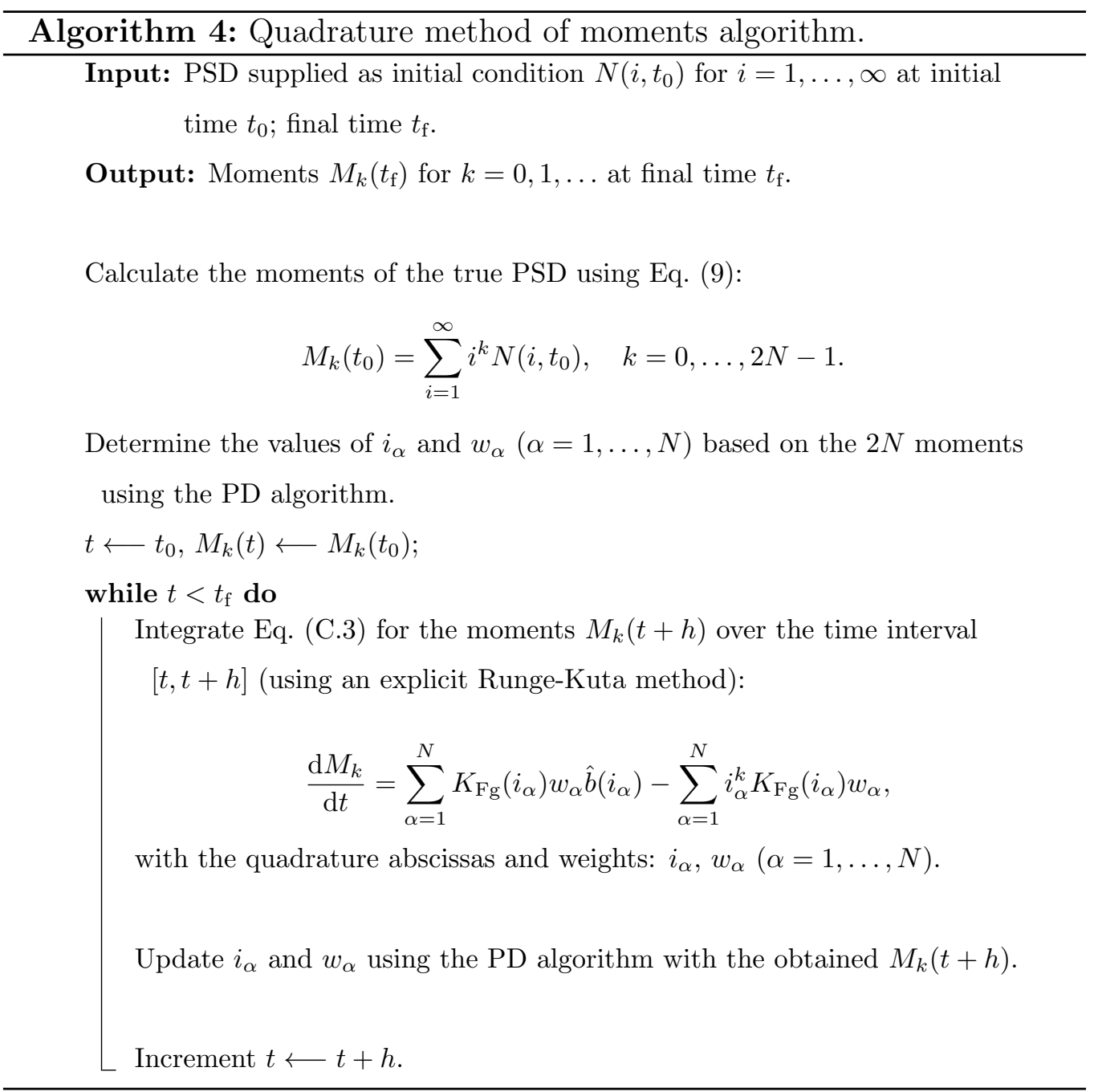


[1] J. Kumar, G. Warnecke, M. Peglow, S. Heinrich, Comparison of numerical methods for solving population balance equations incorporating aggregation and breakage, Powder Technol. 189 (2) (2009) 218-229. doi:10.1016/j.powtec.2008.04.014.

[2] R. B. Diemer, J. H. Olson, A moment methodology for coagulation and breakage problems: part 2-moment models and distribution reconstruction, Chem. Eng. Sci. 57 (12) (2002) 2211-2228. doi:10.1016/S00092509(02)00112-4.

[3] C. A. Sundback, J. M. Beér, A. F. Sarofim, Fragmentation behavior of single coal particles in a fluidized bed, Symp. (Int.) Combust. 20 (1) (1984) 1495-1503. doi:10.1016/S0082-0784(85)80643-3.

[4] S. J. Harris, M. M. Maricq, The role of fragmentation in defining the signature size distribution of diesel soot, J. Aerosol Sci. 33 (6) (2002) 935-942. doi:10.1016/S0021-8502(02)00045-9.

[5] S. Wu, E. K. Y. Yapp, J. Akroyd, S. Mosbach, R. Xu, W. Yang, M. Kraft, A moment projection method for population balance dynamics with a shrinkage term, J. Comput. Phys. Submitted.

[6] M. E. Mueller, G. Blanquart, H. Pitsch, Hybrid method of moments for modeling soot formation and growth, Combust. Flame 156 (6) (2009) 1143-1155. doi:10.1016/j.combustflame.2009.01.025.

[7] M. E. Mueller, G. Blanquart, H. Pitsch, Modeling the oxidation-induced fragmentation of soot aggregates in laminar flames, Proc. Combust. Inst. 33 (1) (2011) 667-674. doi:10.1016/j.proci.2010.06.036. 
[8] T. W. Peterson, Similarity solutions for the population balance equation describing particle fragmentation, Aerosol Sci. Tech. 5 (1) (1986) 93-101. doi:10.1080/02786828608959079.

[9] M. C. Bruns, O. A. Ezekoye, Development of a hybrid sectional quadrature-based moment method for solving population balance equations, J. Aerosol Sci. 54 (2012) 88-102. doi:10.1016/j.jaerosci.2012.07.003.

[10] H. M. Hulburt, S. Katz, Some problems in particle technology: a statistical mechanical formulation, Chem. Eng. Sci. 19 (8) (1964) 555-574. doi:10.1016/0009-2509(64)85047-8.

[11] D. Grosschmidt, H. Bockhorn, M. Goodson, M. Kraft, Two approaches to the simulation of silica particle synthesis, Proc. Combust. Inst. 29 (1) (2002) 1039-1046. doi:10.1016/S1540-7489(02)80131-6.

[12] E. Madadi-Kandjani, A. Passalacqua, An extended quadrature-based moment method with log-normal kernel density functions, Chem. Eng. Sci. 131 (2015) 323-339. doi:10.1016/j.ces.2015.04.005.

[13] J. Akroyd, A. J. Smith, L. R. McGlashan, M. Kraft, Comparison of the stochastic fields method and DQMoM-IEM as turbulent reaction closures, Chem. Eng. Sci. 65 (20) (2010) 5429-5441. doi:10.1016/j.ces.2010.06.039.

[14] J. C. Barrett, N. A. Webb, A comparison of some approximate methods for solving the aerosol general dynamic equation, J. Aerosol Sci. 29 (1-2) (1998) 31-39. doi:10.1016/S0021-8502(97)00455-2. 
[15] A. Falola, A. Borissova, X. Z. Wang, Extended method of moment for general population balance models including size dependent growth rate, aggregation and breakage kernels, Comput. Chem. Eng. 56 (2013) 1-11. doi:10.1016/j.compchemeng.2013.04.017.

[16] G. Madras, B. J. McCoy, Reversible crystal growth-dissolution and aggregation-breakage: numerical and moment solutions for population balance equations, Powder Technol. 143-144 (2004) 297-307. doi:10.1016/j.powtec.2004.04.022.

[17] R. McGraw, Description of aerosol dynamics by the quadrature method of moments, Aerosol Sci. Tech. 27 (2) (1997) 255-265. doi:10.1080/02786829708965471.

[18] D. L. Marchisio, J. T. Pikturna, R. O. Fox, R. Dennis Vigil, A. A. Barresi, Quadrature method of moments for population-balance equations, AIChE J. 49 (5) (2003) 1266-1276. doi:10.1002/aic.690490517.

[19] D. L. Marchisio, R. Dennis Vigil, R. O. Fox, Implementation of the quadrature method of moments in CFD codes for aggregation-breakage problems, Chem. Eng. Sci. 58 (15) (2003) 3337-3351. doi:10.1016/S00092509(03)00211-2.

[20] D. L. Marchisio, R. Dennis Vigil, R. O. Fox, Quadrature method of moments for aggregation-breakage processes, J. Colloid Interface Sci. 258 (2) (2003) 322-334. doi:10.1016/S0021-9797(02)00054-1.

[21] D. L. Marchisio, R. O. Fox, Solution of population balance equations 
using the direct quadrature method of moments, J. Aerosol Sci. 36 (1) (2005) 43-73. doi:10.1016/j.jaerosci.2004.07.009.

[22] S. Kumar, D. Ramkrishna, On the solution of population balance equations by discretization-I. A fixed pivot technique, Chem. Eng. Sci. 51 (8) (1996) 1311-1332. doi:10.1016/0009-2509(96)88489-2.

[23] S. Kumar, D. Ramkrishna, On the solution of population balance equations by discretization-II. A moving pivot technique, Chem. Eng. Sci. 51 (8) (1996) 1333-1342. doi:10.1016/0009-2509(95)00355-X.

[24] F. Gelbard, J. H. Seinfeld, Simulation of multicomponent aerosol dynamics, J. Colloid Interface Sci. 78 (2) (1980) 485-501. doi:10.1016/0021-9797(80)90587-1.

[25] M. J. Hounslow, R. L. Ryall, V. R. Marshall, A discretized population balance for nucleation, growth, and aggregation, AIChE J. 34 (11) (1988) 1821-1832. doi:10.1002/aic.690341108.

[26] M. J. Hounslow, A discretized population balance for continuous systems at steady state, AIChE J. 36 (1) (1990) 106-116. doi:10.1002/aic.690360113.

[27] V. Alopaeus, M. Laakkonen, J. Aittamaa, Solution of population balances with breakage and agglomeration by high-order momentconserving method of classes, Chem. Eng. Sci. 61 (20) (2006) 6732-6752. doi:10.1016/j.ces.2006.07.010.

[28] M. D. Smooke, C. S. McEnally, L. D. Pfefferle, R. J. Hall, M. B. Colket, Computational and experimental study of soot formation in a 
coflow, laminar diffusion flame, Combust. Flame 117 (1-2) (1999) 117139. doi:10.1016/S0010-2180(98)00096-0.

[29] N. A. Eaves, S. B. Dworkin, M. J. Thomson, The importance of reversibility in modeling soot nucleation and condensation processes, Proc. Combust. Inst. 35 (2) (2015) 1787-1794. doi:10.1016/j.proci.2014.05.036.

[30] K. F. Lee, R. I. A. Patterson, W. Wagner, M. Kraft, Stochastic weighted particle methods for population balance equations with coagulation, fragmentation and spatial inhomogeneity, J. Comput. Phys. 303 (2015) 1-18. doi:10.1016/j.jcp.2015.09.031.

[31] M. Kraft, W. Wagner, Numerical study of a stochastic particle method for homogeneous gas-phase reactions, Comput. Math. Appl. 45 (1-3) (2003) 329-349. doi:10.1016/S0898-1221(03)80022-6.

[32] R. I. A. Patterson, W. Wagner, M. Kraft, Stochastic weighted particle methods for population balance equations, J. Comput. Phys. 230 (19) (2011) 7456-7472. doi:10.1016/j.jcp.2011.06.011.

[33] A. Eibeck, W. Wagner, Stochastic interacting particle systems and nonlinear kinetic equations, Ann. Appl. Probab. 13 (3) (2003) 845-889. doi:10.1214/aoap/1060202829.

[34] A. Braumann, M. Kraft, W. Wagner, Numerical study of a stochastic particle algorithm solving a multidimensional population balance model for high shear granulation, J. Comput. Phys. 229 (20) (2010) 7672-7691. doi:10.1016/j.jcp.2010.06.021. 
[35] E. K. Y. Yapp, R. I. A. Patterson, J. Akroyd, S. Mosbach, E. M. Adkins, J. H. Miller, M. Kraft, Numerical simulation and parametric sensitivity study of optical band gap in a laminar coflow ethylene diffusion flame, Combust. Flame 167 (2016) 320-334. doi:10.1016/j.combustflame.2016.01.033.

[36] M. Balthasar, M. Kraft, A stochastic approach to calculate the particle size distribution function of soot particles in laminar premixed flames, Combust. Flame 133 (3) (2003) 289-298. doi:10.1016/S00102180(03)00003-8.

[37] M. Frenklach, Method of moments with interpolative closure, Chem. Eng. Sci. 57 (12) (2002) 2229-2239. doi:10.1016/S0009-2509(02)001136.

[38] M. Strumendo, H. Arastoopour, Solution of PBE by MOM in finite size domains, Chem. Eng. Sci. 63 (10) (2008) 2624-2640. doi:10.1016/j.ces.2008.02.010.

[39] C. Yuan, F. Laurent, R. Fox, An extended quadrature method of moments for population balance equations, J. Aerosol Sci. 51 (2012) 1-23. doi:10.1016/j.jaerosci.2012.04.003.

[40] C. Blumstein, J. C. Wheeler, Modified-moments method: applications to harmonic solids, Phys. Rev. B 8 (1973) 1764-1776. doi:10.1103/PhysRevB.8.1764.

[41] G. H. Golub, Some modified matrix eigenvalue problems, SIAM REV. 15 (1973) 318-334. doi:10.1137/1015032. 
[42] G. H. Press, S. A. Teukolsky, W. T. Vetterling, B. P. Flannery, The art of scientific computing, Cambridge University Press, Cambridge, 2007.

[43] A. Buffo, M. Vanni, D. L. Marchisio, On the implementation of moment transport equations in OpenFOAM: Boundedness and realizability, Int. J. Multiph. Flow. 85 (2016) 223-235. doi:10.1016/j.ijmultiphaseflow.2016.06.017.

[44] D. L. Wright Jr, Numerical advection of moments of the particle size distribution in Eulerian models, J. Aerosol Sci. 38 (2007) 352-369. doi:10.1016/j.jaerosci.2006.11.011.

[45] V. Vikas, Z. J. Wang, A. Passalacqua, R. O. Fox, Realizable high-order finite-volume schemes for quadrature-based moment methods, J. Comput. Phys. 230 (2011) 5328-5352. doi:10.1016/j.jcp.2011.03.038.

[46] D. Kah, F. Laurent, M. Massot, S. Jay, A high order moment method simulating evaporation and advection of a polydisperse liquid spray, J. Comput. Phys. 231 (2012) 394-422. doi:10.1016/j.jcp.2011.08.032.

[47] R. M. Ziff, E. D. McGrady, Kinetics of polymer degradation, Macromolecules 19 (10) (1986) 2513-2519. doi:10.1021/ma00164a010.

[48] E. D. McGrady, R. M. Ziff, "Shattering" transition in fragmentation, Phys. Rev. Lett. 58 (1987) 892-895. doi:10.1103/PhysRevLett.58.892.

[49] R. M. Ziff, E. D. McGrady, The kinetics of cluster fragmentation and depolymerisation, J. Phys. A Math. Gen. 18 (15) (1985) 3027-3037. doi:10.1088/0305-4470/18/15/026. 
648

649

650

651

652

653

654

655

656

657

[50] R. M. Ziff, New solutions to the fragmentation equation, J. Phys. A Math. Gen. 24 (12) (1991) 2821-2828. doi:10.1088/0305$4470 / 24 / 12 / 020$.

[51] M. Kostoglou, A. J. Karabelas, Optimal low order methods of moments for solving the fragmentation equation, Powder Technol. 143-144 (2004) 280-290. doi:10.1016/j.powtec.2004.04.020.

[52] M. Kostoglou, A. J. Karabelas, On the self-similar solution of fragmentation equation: numerical evaluation with implications for the inverse problem, J. Colloid Interface Sci. 284 (2) (2005) 571-581. doi:10.1016/j.jcis.2004.10.029.

[53] R. G. Gordon, Error Bounds in Equilibrium Statistical Mechanics, J. Math. Phys. 9 (1968) 655-663. doi:10.1063/1.1664624. 